\title{
Non-coplanar Model States in Quantum Magnetism Applications of the High-Order Coupled Cluster Method
}

\author{
D. J. J. Farnell ${ }^{1}$ D $\cdot$ R. F. Bishop ${ }^{2,3} \cdot$ J. Richter ${ }^{4,5}$
}

Received: 17 October 2018 / Accepted: 12 April 2019 / Published online: 2 May 2019

(c) The Author(s) 2019

\begin{abstract}
Coplanar model states for applications of the coupled cluster method (CCM) to problems in quantum magnetism are those in which all spins lie in a plane, whereas three-dimensional (3D) model states are, by contrast, non-coplanar ones in which all the spins do not lie in any single plane. A crucial first step in applying the CCM to any such lattice quantum spin system is to perform a passive rotation of the local spin axes so that all spins in the model state appear mathematically to point in the same (say, downwards $z$-)direction. Whereas this process leads to terms with only real coefficients in the rotated Hamiltonian for coplanar model states, an additional complication arises for 3D model states where the corresponding coefficients can become complex-valued. We show here for the first time how high-order implementations of the CCM can be performed for such Hamiltonians. We explain in detail why the extension of the computational implementation of the CCM when going from coplanar to 3D model states is a non-trivial task that has not hitherto been undertaken. To illustrate these new developments, we present results for three cases: (a) the spin-half one-dimensional Ising ferromagnet in an applied transverse magnetic field (as an exactly solvable test model to use as a yardstick for the viability and accuracy of our new methodology); (b) the spinhalf triangular-lattice Heisenberg antiferromagnet in the presence of an external magnetic field; and (c) the spin- $S$ triangular-lattice $X X Z$ antiferromagnet in the presence of an external magnetic field, for the cases $\frac{1}{2} \leq S \leq 5$. For 3D model states the sets of algebraic CCM equations for the ket- and bra-state correlation coefficients become complex-valued, but ground-state expectation values of all physical observables are manifestly real numbers, as required, and as we explicitly demonstrate in all three applications. Indeed, excellent correspondence is seen with the results of other methods, where they exist, for these systems. In particular, our CCM results demonstrate explicitly that coplanar ordering is favoured over non-coplanar ordering for the triangular-lattice spin-half Heisenberg antiferromagnet at all values of the applied external magnetic field, whereas for the anisotropic $X X Z$ model noncoplanar ordering can be favoured in some regions of the parameter space. Specifically, we present a precise determination of the boundary (i.e., the critical value of the $X X Z$ anisotropy parameter $\Delta$ ) between a 3D ground state and a coplanar ground state for the $X X Z$ model for
\end{abstract}

Communicated by Alessandro Giuliani.

$凶 \quad$ D. J. J. Farnell

FarnellD@cardiff.ac.uk

Extended author information available on the last page of the article 
values for the external magnetic field near to saturation, for values of the spin quantum number $S \leq 5$. Although the CCM calculations are computationally intensive for this frustrated model, especially for high spin quantum numbers, our accurate new results certainly improve our understanding of it.

Keywords Quantum magnetism · Coupled cluster method · Computational simulation

\section{Introduction}

The coupled cluster method (CCM) [1-15] is a powerful method of quantum many-body theory that has long been used to study strongly interacting and highly frustrated quantum spin systems with great success [16-42]. The introduction of the "high-order" CCM [21-23] for these systems has led to a step-change in its accuracy. The high-order CCM employs very high orders of approximation schemes, for which the equations that determine all multispin correlations retained at any given level are both derived and subsequently solved by using massively parallel computational tools $[21-23,43]$. The CCM is now fully competitive with the best of other approximate methods, especially for systems of $N$ spins on the sites of a lattice in two (see, e.g., [33,37,38,42] and references cited therein) or three (see, e.g., [39,40] and references cited therein) spatial dimensions. Unlike several other approximate quantum many-body methods that are limited in their range of applicability by frustration (i.e., where bonds in the Hamiltonian compete against each other to achieve energy minimisation), the CCM has been applied previously even to highly frustrated and strongly correlated quantum spin systems with much success. Thus, recently it has been demonstrated, for example, that the high accuracy needed to investigate the quantum ground-state selection of competing states of the kagome antiferromagnet is provided by high-order CCM calculations [33,38,39]. Another advantage of the high-order CCM is that it is very flexible. For example, both in principle and in practice, the CCM technique can treat essentially all Hamiltonians containing either single spin operators and/or products of two spin operators, on any crystallographic lattice, and for any spin quantum number $S$. We note too that, unlike most alternative techniques, the CCM can be applied from the outset in the thermodynamic limit, $N \rightarrow \infty$, at every level of approximate implementation, thereby obviating the need for any finite-size scaling of the results.

In all practical implementations of the CCM the many-body correlations present in the exact (ground or excited) state of the system under investigation are expressed with respect to a suitable model (or reference) state, as we explain in more detail below in Sect. 2. Coplanar model spin states used in the CCM are those states in which all spins lie in a plane, whereas three-dimensional (3D) model spin states are non-coplanar states in which the spins do not lie in any one plane. We remark that, until now, only coplanar model spin states have been used in all prior CCM calculations in the field of quantum magnetism for reasons that we now explain.

Thus, an important ingredient used in all practical applications of the CCM to spin-lattice systems [16-42] is to rotate the local spin axes of all spins in the model state such that they appear (mathematically only) to be point in the "downwards" $z$-direction. One can always choose a set of rotations that leads to terms in the Hamiltonian that contain only real-valued coefficients, with respect to the new set of local spin axes, for the coplanar model states. By contrast, three-dimensional (3D) (non-coplanar) model states inevitably lead to terms in the new Hamiltonian after rotation of the local spin axes that contain complex-valued coefficients. These cases are more difficult to treat both analytically and computationally. Of course, all 
macroscopic physical parameters calculated within the CCM, such as the ground-state energy and magnetic order parameter, still have to be real numbers because the transformations of local spin axes are unitary and the resulting Hamiltonian is still Hermitian. Nevertheless, the intervening multispin correlation coefficients are necessarily complex-valued quantities.

In this article, we explain how we can carry out CCM calculations for such Hamiltonians that contain terms in the Hamiltonian after rotation of local spin axes with complex-valued coefficients. We show that the amendments to the existing CCM code for spin-lattice models [43] to be able to treat such cases is non-trivial. In order to illustrate the new technique, we present three separate applications to models of considerable interest in quantum magnetism. As a first test of the new methodology we present results in Sect. 3 for the exactly solvable one-dimensional Ising model in a transverse external magnetic field, and an explicit analytical calculation of the lowest-order implementation of the CCM is presented in detail for this model in Appendix A. Secondly, in Sect. 4 we then describe results for the spin-half Heisenberg model on the triangular lattice at zero temperature in the presence of an external magnetic field, in which we make explicit use of the 3D "umbrella" state as our CCM model state, and an explicit derivation of the Hamiltonian after the rotations of the local spin axes for this state is presented in Appendix B. Lastly, in Sect. 5, the phase diagram of the spin-half $X X Z$ model on the triangular lattice at zero temperature, also in the presence of an external magnetic field (near saturation), is examined. Here we again employ the 3D "umbrella" state as a possible CCM model state, and we show how its use now leads to an improved quantitative description and understanding of this model. We conclude with a brief summary of our results in Sect. 6.

\section{The Coupled Cluster Method (CCM)}

\subsection{Ground-State Formalism}

As the methodology of the CCM has been discussed extensively elsewhere [1-42], only a brief overview of the method is presented here. The ground-state Schrödinger equations are given by

$$
\hat{H}|\Psi\rangle=E_{g}|\Psi\rangle ; \quad\langle\tilde{\Psi}| \hat{H}=E_{g}\langle\tilde{\Psi}|,
$$

in terms of the Hamiltonian $\hat{H}$, and where formally, for normalisation, we require

$$
\langle\tilde{\Psi}|=\frac{(|\Psi\rangle)^{\dagger}}{\langle\Psi \mid \Psi\rangle} .
$$

The bra and ket states for our $N$-spin system (with each of the spins carrying the spin quantum number $S$ ) are parametrised independently in the forms

$$
\begin{aligned}
& |\Psi\rangle=\mathrm{e}^{\bar{S}}|\Phi\rangle ; \quad \bar{S}=\sum_{I \neq 0} \mathcal{S}_{I} \hat{C}_{I}^{+}, \\
& \langle\tilde{\Psi}|=\langle\Phi| \hat{\tilde{S}} \mathrm{e}^{-\bar{S}} ; \quad \hat{\tilde{S}}=1+\sum_{I \neq 0} \tilde{\mathcal{S}}_{I} \hat{C}_{I}^{-},
\end{aligned}
$$

within the normal coupled cluster method, in terms of the multi-configurational CCM (creation and destruction) correlation operators, $\hat{S}$ and $\hat{\tilde{S}}$, respectively. The index $I$ here is a set-index that denotes a set of lattice sites, $I=\left\{i_{1}, i_{2}, \ldots, i_{n} ; n=1,2, \ldots 2 S N\right\}$, in which each site may appear no more than $2 S$ times, for reasons we describe below. We shall be 
interested specifically in the case of infinite systems, $N \rightarrow \infty$. Note that $\hat{C}_{0}^{+} \equiv \hat{\mathbb{1}}$ is defined to be the identity operator in the many-body Hilbert space, the operators $\hat{C}_{I}^{+}$and $\hat{C}_{I}^{-} \equiv\left(\hat{C}_{I}^{+}\right)^{\dagger}$ are respectively, $\forall I \neq 0$, multispin creation and destruction operators, for clusters of up to $N$ spins, which are defined more fully below, and $\mathcal{S}_{I}$ and $\tilde{\mathcal{S}}_{I}$ are the CCM ground-state ketand bra-state ( $c$-number) multispin correlation coefficients, respectively. We use model states (denoted $|\Phi\rangle$ for the ket state and $\langle\Phi|$ for the bra state) as references states for the CCM. The ket state $|\Phi\rangle$ is required to be a fiducial vector (or cyclic vector) with respect to the complete set of mutually commuting, multispin creation operators $\left\{\hat{C}_{I}^{+}\right\}$. Equivalently, the set of states $\left\{\hat{C}_{I}^{+}|\Phi\rangle\right\}$ is a complete basis for the ket-state Hilbert space. Furthermore, $|\Phi\rangle$ is also defined to be a generalised vacuum state with respect to the set of operators $\left\{\hat{C}_{I}^{+}\right\}$, in the sense that

$$
\left\langle\Phi\left|\hat{C}_{I}^{+}=0=\hat{C}_{I}^{-}\right| \Phi\right\rangle ; \quad \forall I \neq 0 .
$$

We note that, with these conditions fulfilled, the exact ground-state ket- and bra-state wave functions, $|\Psi\rangle$ and $\langle\tilde{\Psi}|$, respectively, now satisfy the normalisation conditions

$$
\langle\tilde{\Psi} \mid \Psi\rangle=\langle\Phi \mid \Psi\rangle=\langle\Phi \mid \Phi\rangle \equiv 0 .
$$

We now define the ground-state energy functional, $\bar{H} \equiv\langle\tilde{\Psi}|\hat{H}| \Psi\rangle=\left\langle\Phi\left|\hat{\tilde{S}} \mathrm{e}^{-\hat{S}} \hat{H} \mathrm{e}^{\hat{S}}\right| \Phi\right\rangle$, such that the CCM ket- and bra-state equations are given by extremising $\bar{H}$ with respect to all of the CCM multispin correlation coefficients,

$$
\begin{gathered}
\frac{\partial \bar{H}}{\partial \tilde{\mathcal{S}}_{I}}=0 \Rightarrow\left\langle\Phi\left|\hat{C}_{I}^{-} \mathrm{e}^{-\hat{S}_{H}} H \mathrm{e}^{\hat{S}}\right| \Phi\right\rangle=0, \quad \forall I \neq 0, \\
\frac{\partial \bar{H}}{\partial \mathcal{S}_{I}}=0 \quad \Rightarrow \quad\left\langle\Phi\left|\hat{\tilde{S}}^{-\hat{S}}\left[\hat{H}, \hat{C}_{I}^{+}\right] \mathrm{e}^{\hat{S}}\right| \Phi\right\rangle=0 . \quad \forall I \neq 0 .
\end{gathered}
$$

With these equations satisfied, the CCM ground-state energy is now given by

$$
E_{g}=\left\langle\Phi\left|\mathrm{e}^{-\hat{S}} \hat{H} \mathrm{e}^{\hat{S}}\right| \Phi\right\rangle
$$

Equation (9) is a function of the ket-state correlation coefficients $\left\{\mathcal{S}_{I}\right\}$ only and it involves the similarity transform, $\mathrm{e}^{-\hat{S}} \hat{H} \mathrm{e}^{\hat{S}}$, of $\hat{H}$, which is a key feature of any CCM calculation. We may evaluate this expression in terms of the well-known nested-commutator expansion for the similarity transform of an arbitrary operator $\hat{O}$,

$$
\mathrm{e}^{-\hat{S}} \hat{O} \mathrm{e}^{\hat{S}}=\hat{O}+[\hat{O}, \hat{S}]+\frac{1}{2 !}[[\hat{O}, \hat{S}], \hat{S}]+\cdots
$$

The Hamiltonian, $\hat{H}$, like any other physical operator whose CCM ground-state expectation value we wish to calculate, normally contains only finite sums of products of spin operators, and so their nested-commutator expansions of Eq. (10) generally terminate after a finite number of terms.

The choice of model state depends on the specific details of the model under consideration and so this is discussed in detail below. However, we remark that a passive rotation of the local spin axes is used in all cases such that all spins point in the negative $z$-direction after rotation of the local spin axes. This process allows us to treat all spins equivalently and it simplifies the mathematical formulation of the CCM and the subsequent derivation of its basic equations, viz., Eqs. (7) and (8), very considerably. The corresponding multispin creation 
operators $\left\{\hat{C}_{I}^{+}\right\}$are thus defined with respect to this CCM model state, such that

$$
|\Phi\rangle=\bigotimes_{k=1}^{N}|\downarrow\rangle_{i_{k}} ; \quad \hat{C}_{I}^{+}=\hat{s}_{i_{1}}^{+} \hat{s}_{i_{2}}^{+} \ldots \hat{s}_{i_{n}}^{+}, n=1,2, \ldots, 2 S N,
$$

in these rotated local spin-space frames, where $i_{k}$ denotes an arbitrary lattice site, $|\downarrow\rangle_{i_{k}}$ is the "downward-pointing" state of a spin on site $i_{k}$ with spin quantum number $S$ (i.e., defined so that $\hat{s}_{i_{k}}^{z}|\downarrow\rangle_{i_{k}}=-S|\downarrow\rangle_{i_{k}}$ ), and $\hat{s}_{i_{k}}^{+} \equiv \hat{s}_{i_{k}}^{x}+\mathrm{i} \hat{s}_{i_{k}}^{y}$ is the usual SU(2) spin-raising operator on site $i_{k}$.

The CCM formalism would be exact if all possible multispin cluster correlations could be included in the operators $\hat{S}$ and $\hat{\tilde{S}}$. However, this is normally impossible to achieve practically. In most cases, systematic approximation schemes are used to truncate the respective summations in Eqs. (3) and (4) for these operators, by restricting the sets of multispin configurations $\{I\}$ to some manageable subset within some hierarchical scheme that becomes exact in the limit that all configurations are retained. In the present paper we use two schemes that are denoted as the SUB $n-n$ and LSUB $n$ schemes, respectively. The more general SUB $n-$ $m$ scheme retains all correlations involving only $n$ or fewer spin flips (with respect to the respective model state $|\Phi\rangle$ ) that span a range of no more than $m$ contiguous lattice sites. By contrast, in the localised LSUB $n$ scheme all multispin correlations over all distinct locales on the lattice defined by $n$ or fewer contiguous sites are retained. Each spin flip is defined to require the action of a spin-raising operator $\hat{s}_{i_{n}}^{+}$acting just once, and a set of lattice sites is said to be contiguous if every site of the set is a nearest neighbour (in some specified lattice geometry) to at least one other member of the set. The LSUB $n$ and SUB $n-n$ schemes are thus identical only for the limiting case when $S=1 / 2$. For higher spin quantum numbers $S$, the LSUB $m$ scheme is equivalent to the SUB $n-m$ scheme if and only if $n=2 S m$. Spincluster configurations $I$ that are equivalent under the space- and point-group symmetries of the crystallographic lattice (as well as of both the Hamiltonian and the model state under consideration) are counted only once by explicitly incorporating those symmetries into the calculation, and these clusters are referred to as "fundamental clusters". The number of such fundamental clusters used for the ground-state expansions for $|\Psi\rangle$ and $\langle\tilde{\Psi}|$ at the respective $n$ th-order level of (either LSUBn or SUB $n-n$ ) approximation to Eqs. (3) and (4) is denoted by $N_{f}(n)$.

Although, formally, the CCM correlation operators $\hat{S}$ and $\hat{\tilde{S}}$ of Eqs. (3) and (4) must obey the condition

$$
\langle\Phi| \hat{\tilde{S}}=\frac{\langle\Phi| \mathrm{e}^{\hat{S}^{\dagger}} \mathrm{e}^{\hat{S}}}{\left\langle\Phi\left|\mathrm{e}^{\hat{S}^{\dagger}} \mathrm{e}^{\hat{S}}\right| \Phi\right\rangle},
$$

which is implied by Hermiticity, in practice this may not be exactly fulfilled at finite levels of (LSUB $n$ or $\mathrm{SUB} n-n$ ) approximate implementation, due to the independent parametrisations of the two operators. However, this minor drawback of the CCM is far outweighed in practice by the two huge advantages that the method exactly obeys both the Goldstone linked-cluster theorem and the very important Hellmann-Feynman theorem at all levels in the approximation hierarchies. The former implies that we can work from the outset in the required thermodynamic limit of an infinite number of spins, $N \rightarrow \infty$, while the latter implies that the expectation values of all physical parameters are calculated within the CCM on the same footing as the energy and in a fully self-consistent manner.

Unlike in many other competing formulations of quantum many-body theory, the CCM thus never needs any finite-size scaling of the results obtained with it. Indeed, the sole approximation that is ever made within any application of the CCM is to extrapolate the 
results obtained for any physical parameter within the (LSUB $n$ or SUB $n-n$ ) approximation hierarchy used to the limit $n \rightarrow \infty$ where the method becomes exact. By now, a great deal of experience has been acquired on how to perform such CCM extrapolations, and we allude here to one such calculation in Sect. 3, and invite the reader to consult the literature cited above for further details.

\subsection{Computational Aspects for 3D Model States}

The CCM equations (7) and (8) may be readily derived and solved analytically at low orders of approximation. A full explanation of how this is carried out for the LSUB1 approximation for the spin-half ferromagnetic Ising chain in a transverse magnetic field, which we study in Sect. 3 is given in Appendix A. Highly intensive computational methods [21-23] are essential at higher orders of LSUB $n$ or $\mathrm{SUB} n-n$ approximation because the number $N_{f}(n)$ of fundamental clusters (and so therefore also the computational resources necessary to store and solve them) scales approximately exponentially with the order $n$ of the approximation scheme being used. There are four distinct steps to perform in carrying out high-order CCM calculations for the ground state for "3D model states," each of which has a counterpart in the "standard" CCM code [43] that pertains only to coplanar states. (As a short-hand only, we shall refer to any case that results in the Hamiltonian containing terms with coefficients that are complex-valued after rotation of the local spin axes to be a "3D model state," although clearly these are some essentially artificial cases, e.g., the transverse Ising model presented below, where the model state might be coplanar.)

The first step is to read in "CCM script files" that define the basic problem to be solved. We remark that the derivation of Hamiltonians after rotation of local spin axes for the 3D model states is non-trivial because we must carry out at least two sets of rotations. An example of this process is given for the spin-half triangular-lattice Heisenberg model in the presence of an external magnetic field in Appendix B. As a consequence the resulting CCM script file is much longer than for coplanar model states because we now have terms in the Hamiltonian with both real and imaginary coefficients.

The second step involves the enumeration of all connected clusters (also called "lattice animals") and all disconnected clusters that are to be retained at a given level of LSUB $n$ or $\mathrm{SUB} n-n$ approximation for a given lattice and spin quantum number $S$ that are distinct under the lattice, model state and Hamiltonian symmetries (and perhaps that also satisfy some such conservation rule as $s_{T}^{z}=0$, where $\hat{s}_{T}^{z} \equiv \sum_{i=1}^{N} \hat{s}_{i}^{z}$, which would pertain, for example, to all models whose Hamiltonians contain only spins interacting pairwise via isotropic Heisenberg exchange interactions). This step is no more difficult for 3D model states than for coplanar model states, although clearly this step is itself highly non-trivial to perform computationally.

The third step involves deriving and storing the basic CCM ground-state equations. In order to find these equations, we first partition the multispin cluster configuration pertaining to the set index $I$ for the operator $\hat{C}_{I}^{-}$in the ket-state equation $\left\langle\Phi\left|\hat{C}_{I}^{-} \mathrm{e}^{-\hat{S}} \hat{H} \mathrm{e}^{\hat{S}}\right| \Phi\right\rangle=0$ of Eq. (7) into the products of "high-order CCM operators" [21-23]. There are a huge number of partitions potentially and each term in a new potential contribution to the ket-state equations must be tested for suitability, i.e., all subclusters are checked against a list of fundamental clusters after any appropriate space- and point group symmetries (plus any applicable conservation laws) have been employed. This is arguably the most difficult step in carrying out any highorder CCM calculation, and effectively we must run this code twice for the 3D model states: once for the terms in the Hamiltonian with real coefficients and again for the terms with imaginary coefficients. 
The fourth step is to solve the ground-state ket and bra equations and to obtain the groundstate expectation values. For 3D model states, complex-number algebra must be implemented for all subroutines that solve the ket- and bra-state equations (solved by "direct iteration" for 3D model states), and also in those subroutines that determine expectation values such as the ground-state energy of Eq. (9) or other expectation values (i.e., $\bar{A}=\left\langle\Phi\left|\hat{\tilde{S}} e^{-\hat{S}} \hat{A} e^{\hat{S}}\right| \Phi\right\rangle$ ). This is achieved by using options in the $\mathrm{C}++$ compiler.

Although the process of updating the existing high-order CCM code [43] for coplanar states so as to be able now also to utilise 3D model states (resulting in Hamiltonians with terms involving complex-valued coefficients) is therefore straightforward in principle, this process is actually considerably less so in practice because the CCM code [43] itself is extensive and complex. In order to validate the new code, we show in Sect. 3 that analytical low-order LSUB 1 results derived in Appendix A for the transverse Ising model are replicated by the new 3D CCM code. Similarly, for the same model, for higher orders of LSUB $n$ approximation with $n \leq 12$, we also show that the new code exactly replicates the corresponding results obtained using the "standard" code [43]. Both of these results are excellent tests of the new code. Furthermore, all results for each of the three models considered in Secs. 3, 4 and 5 are in excellent agreement with the results of other methods (where they exist). Finally, we remark again that the creation of the CCM script files is more complicated for 3D model states than for coplanar model states.

\section{Spin-Half Ising Ferromagnetic Chain in a Transverse External Magnetic Field}

We take as a first example to demonstrate the feasibility and the accuracy of the new CCM approach an exactly solvable model, namely the one-dimensional (1D) spin- $\frac{1}{2}$ Ising ferromagnet in a transverse magnetic field [44]. The corresponding Hamiltonian is given by

$$
\hat{H}=-\sum_{k=1}^{N} \hat{s}_{k}^{z} \hat{s}_{k+1}^{z}-\lambda \sum_{k=1}^{N} \hat{s}_{k}^{x}=-\sum_{k=1}^{N} \hat{s}_{k}^{z} \hat{s}_{k+1}^{z}-\frac{\lambda}{2} \sum_{k=1}^{N}\left(\hat{s}_{k}^{+}+\hat{s}_{k}^{-}\right),
$$

where the index $k$ runs over all lattice sites on the linear chain (with site $N+1$ equivalent to site 1) and $\hat{s}_{k}^{ \pm} \equiv \hat{s}_{k}^{x} \pm \mathrm{i} \hat{s}_{k}^{y}$. The strength of the applied external transverse magnetic field is given by $\lambda$. Clearly, in the case $\lambda=0$ with no field applied, the spins are ferromagnetically aligned along the $z$-direction. Similarly, for high enough values of $\lambda$ it is clear that the spins will align along the transverse ( $x$-)direction. The CCM model state that we choose for this system is one in which all spins point in the downwards $z$-direction. Thus, the model state is expected to be better for low values of $\lambda$, particularly those below the phase transition that separates the two regimes where the spins are respectively canted to align along some intermediate direction between the $z$ - and $x$-directions (at low values of $\lambda$ ) and fully aligned in the transverse field $(x$-)direction (at high values of $\lambda$ ).

Classically, the spins are canted at an angle $\alpha$ from the (say, downwards) $z$-direction in the presence of the transverse magnetic field $\lambda$. It is trivial to see that the classical ground-state energy $E_{g}^{\mathrm{cl}}$ is minimised for $\alpha=\sin ^{-1} \lambda$ for $\lambda \leq 1$. There is then a classical phase transition at $\lambda=\lambda_{c}^{\mathrm{cl}} \equiv 1$, such that for $\lambda \geq \lambda_{c}^{\mathrm{cl}}$, the spins are all aligned in the direction of the transverse field, with $\alpha=\frac{1}{2} \pi$. We thus have that the classical ground-state energy per spin is given by

$$
\frac{E_{g}^{\mathrm{cl}}}{N}= \begin{cases}-\frac{1}{4}\left(1+\lambda^{2}\right) ; & \lambda \leq 1 \\ -\frac{1}{2} \lambda ; & \lambda \geq 1 .\end{cases}
$$




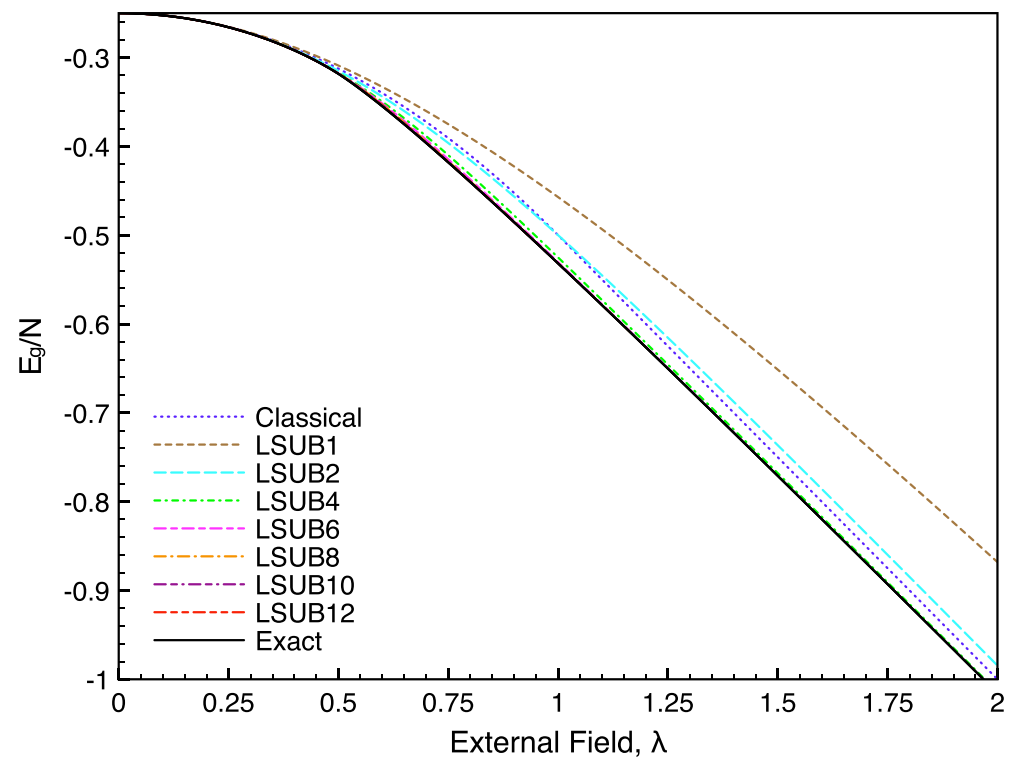

Fig. 1 CCM results for the ground-state energy per spin of the Ising model on the linear chain as a function of the transverse external magnetic field strength, $\lambda$, at various LSUB $n$ levels of approximation. Also shown are the corresponding classical result of Eq. (14) and the exact result [44] of Eq. (17)

Similarly, the classical values of the magnetisations in the $z$-direction (i.e., the Ising direction), $M^{z}$, and in the transverse $x$-direction (i.e., the field direction), $M^{\text {trans. }}$, are trivially found to be given by

$$
M_{\mathrm{cl}}^{z}= \begin{cases}\frac{1}{2} \sqrt{1-\lambda^{2}} ; & \lambda \leq 1 \\ 0 ; & \lambda \geq 1\end{cases}
$$

and

$$
M_{\mathrm{cl}}^{\text {trans. }}= \begin{cases}\frac{1}{2} \lambda ; & \lambda \leq 1 \\ \frac{1}{2} ; & \lambda \geq 1 .\end{cases}
$$

The quantum spin- $\frac{1}{2}$ version of the model can be exactly solved [44]. Thus we also have available to us the corresponding exact expressions for the classical parameters given above in Eqs. (14)-(16), against which we can compare our CCM results. Particular interest attaches to the model due to the fact that the classical phase transition at $\lambda=\lambda_{c}^{\mathrm{cl}} \equiv 1$ is now shifted to the point $\lambda=\lambda_{c} \equiv \frac{1}{2}$. The exact ground-state energy per spin is given by [44]

$$
\frac{E_{g}}{N}=-\frac{1}{4 \pi} \int_{0}^{\pi} \mathrm{d} k \sqrt{1+4 \lambda \cos k+4 \lambda^{2}}
$$

which expression is nonanalytic at the quantum phase transition point $\lambda_{c}=\frac{1}{2}$. It is simple to check that in the two extremes $\lambda \rightarrow 0$ and $\lambda \rightarrow \infty$, Eq. (17) reduces respectively to the two limiting values, $E_{g}(\lambda=0) / N=-\frac{1}{4}$ and $E_{g}(\lambda \rightarrow \infty) / N \rightarrow-\frac{1}{2} \lambda$, exactly as for the classical case given by Eq. (14). This is also just as expected, since in these two limits the fully aligned ferromagnetic states are also eigenstates of the quantum Hamiltonian. Precisely at the quantum phase transition point, Eq. (17) yields the value $E_{g}\left(\lambda=\frac{1}{2}\right) / N=-\frac{1}{\pi}$. The classical result of Eq. (14) is compared with its exact counterpart of Eq. (17) in Fig. 1. 


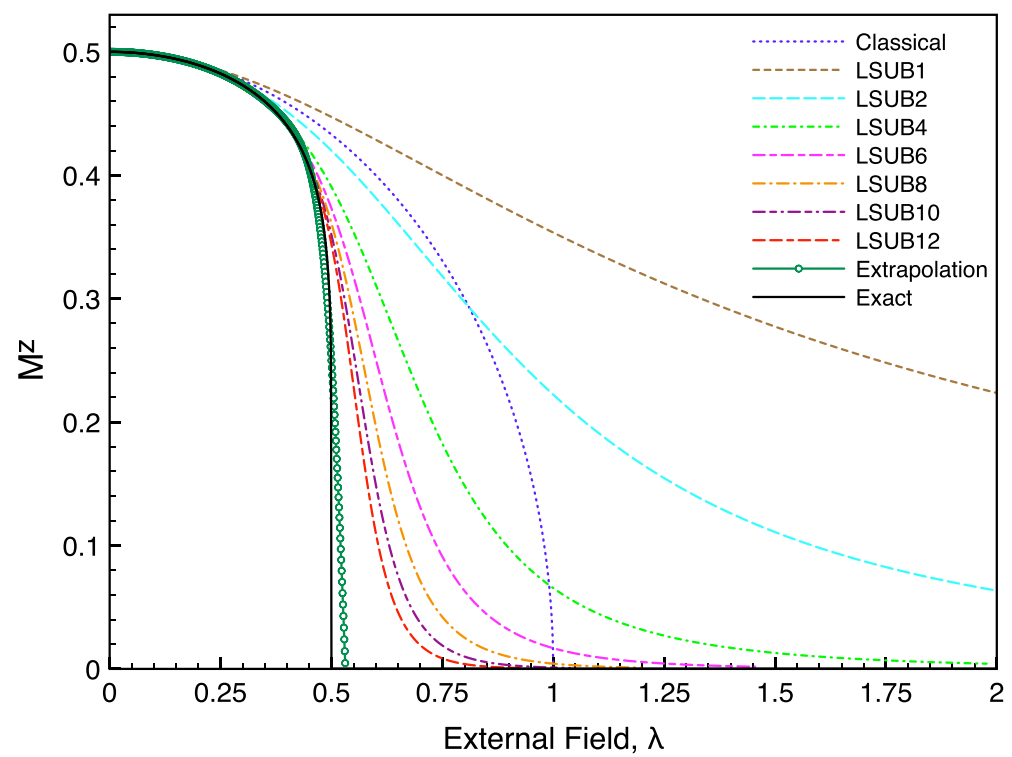

Fig. $2 \mathrm{CCM}$ results for the magnetisation $M^{z}$ of the Ising model on the linear chain as a function of the transverse external magnetic field strength, $\lambda$, at various LSUB $n$ levels of approximation, together with the extrapolation based on all LSUB $n$ results (for both even and odd values of $n$ ) with $6 \leq n \leq 12$, as explained in the text. Also shown are the corresponding classical result of Eq. (15) and the exact result [44] of Eq. (18)

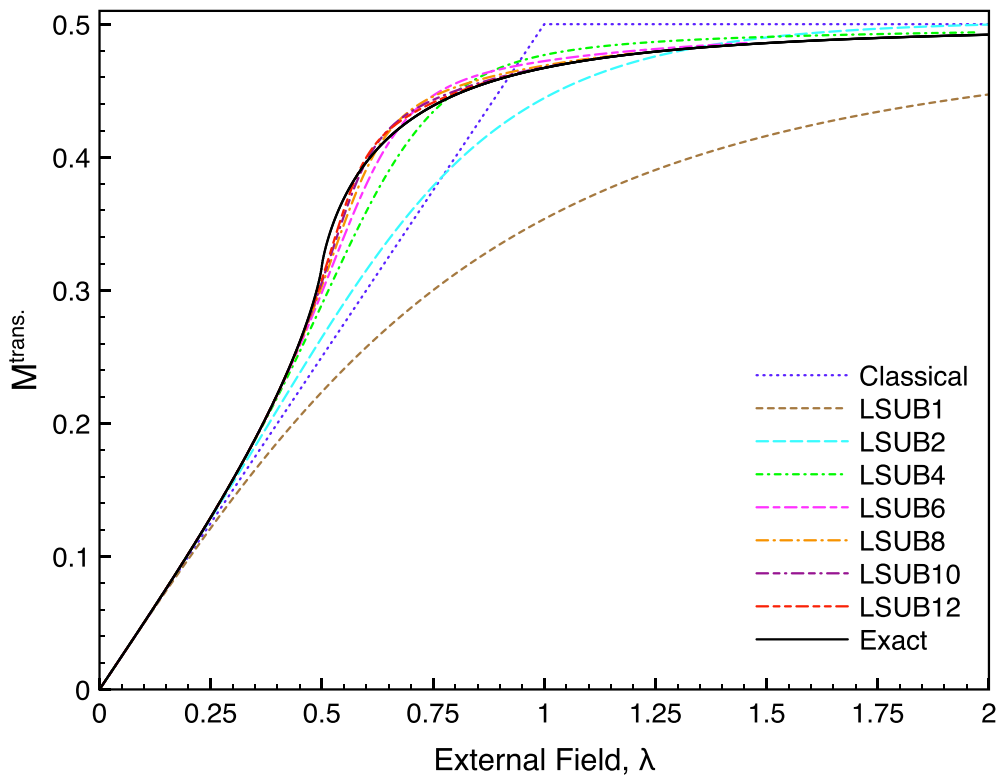

Fig. $3 \mathrm{CCM}$ results for the transverse magnetisation, $M^{\text {trans. }}$, of the Ising model on the linear chain as a function of the transverse external magnetic field strength, $\lambda$, at various LSUB $n$ levels of approximation. Also shown are the corresponding classical result of Eq. (16) and the exact result [44] of Eq. (19) 
The corresponding exact result for the magnetisation in the (Ising) $z$-direction, $M^{z}$, is given by [44]

$$
M^{z}= \begin{cases}\frac{1}{2}\left(1-4 \lambda^{2}\right)^{1 / 8} ; & \lambda \leq \frac{1}{2} \\ 0 ; & \lambda \geq \frac{1}{2},\end{cases}
$$

which now exhibits the phase transition at $\lambda=\lambda_{c} \equiv \frac{1}{2}$ much more clearly than Eq. (17) for the ground-state energy. Once again, the classical and exact results for $M^{z}$, from Eqs. (15) and (18) respectively, are compared in Fig. 2. Finally, the exact result for the transverse magnetisation (i.e., in the field direction) is given by [44]

$$
M^{\text {trans. }}=\frac{1}{2 \pi} \int_{0}^{\pi} \mathrm{d} k \frac{(\cos k+2 \lambda)}{\sqrt{1+4 \lambda \cos k+4 \lambda^{2}}},
$$

which is again nonanalytic at the quantum phase transition point, $\lambda_{c}=\frac{1}{2}$, where it takes the value $M^{\text {trans. }}\left(\lambda=\frac{1}{2}\right)=\frac{1}{\pi}$. It is easy to confirm that when $\lambda$ varies from zero to $\infty, M^{\text {trans. }}$ from Eq. (19) varies smoothly from zero to $\frac{1}{2}$, as shown in Fig. 3 where it is also compared to its classical counterpart of Eq. (16).

For present purposes we now wish to illustrate how the CCM can be applied when we carry out a unitary transformation of the local spin axes that leads to terms in the Hamiltonian with complex-valued coefficients. We use the unitary rotation of the local spin axes (now for all sites $k$ on the linear chain) given by

$$
\hat{s}_{k}^{x} \rightarrow \hat{s}_{k}^{y} ; \quad \hat{s}_{k}^{y} \rightarrow-\hat{s}_{k}^{x} ; \quad \hat{s}_{k}^{z} \rightarrow \hat{s}_{k}^{z},
$$

which simply is equivalent to rotating the transverse field from the $x$ - to the $y$-direction, while leaving the spins aligned in the (negative) $z$-direction. That leads to an alternative representation of the model given by the Hamiltonian

$$
\hat{H}=-\sum_{k=1}^{N} \hat{s}_{k}^{z} \hat{s}_{k+1}^{z}-\lambda \sum_{k=1}^{N} \hat{s}_{k}^{y}=-\sum_{k=1}^{N} \hat{s}_{k}^{z} \hat{s}_{k+1}^{z}-\frac{i \lambda}{2} \sum_{k=1}^{N}\left(\hat{s}_{k}^{-}-\hat{s}_{k}^{+}\right),
$$

where the term with an imaginary coefficient now appears in the transverse external field part of the Hamiltonian. However, we remark again that the eigenvalue spectrum for this Hamiltonian should not change compared to that for the Hamiltonian of Eq. (13). Note too that this rotation of the spins in the $x y$-plane does not affect the model state for this system, namely, one in which all spins point in the downwards $z$-direction.

CCM LSUB1 calculations for both Hamiltonians of Eqs. (13) and (21) are carried out explicitly and independently in Appendix A. Calculations based on the Hamiltonian of Eq. (13) lead to ket- and bra-state correlation coefficients that are real numbers only, whereas those calculations based on the Hamiltonian of Eq. (21) lead to ket- and bra-state correlation coefficients that are complex (i.e., that contain both real and imaginary components). Results for the ground-state energy per spin, $E_{g} / N$, and the magnetisations, $M^{z}$ in the Ising $(z)$ direction and $M^{\text {trans. }}$ in the transverse $(x)$-direction, based on the Hamiltonians of Eqs. (13) and (21) are found to be identical (and so also "real-valued") at the LSUB1 level of approximation, as required. These analytical results provide a preliminary test of the validity of the CCM method for unitary rotations of local spin axes that lead to terms in the Hamiltonian with both real and imaginary coefficients.

The new code developed here for "3D model states" can be applied to the Hamiltonian of Eq. (21) to high orders of LSUBn approximation. These results can be compared to those from the "standard" CCM code [43] that works for coplanar states only, which can be applied 
to the Hamiltonian of Eq. (13). Results from these two codes are again found to agree exactly with each other at equivalent levels of approximation and specifically also with the analytical LSUB1 results presented in Appendix A. The results for the ground-state energy are shown in Fig. 1 and the results for the magnetisations $M^{z}$ and $M^{\text {trans. }}$ are shown in Figs. 2 and 3, respectively. Despite the fact that the ket- and bra-states correlation coefficients are found to be complex-valued for all values of $\lambda(>0)$, the ground-state energies and magnetisations are again found to be real at all approximation levels and for all values of $\lambda$ for the Hamiltonian of Eq. (21) using the new code.

We note that convergence of the LSUBn sequences of approximants becomes worse for larger values of $\lambda(\gtrsim 0.5$ ), exactly as expected, since this region is precisely where the model state becomes a poorer starting point for the CCM calculations, due to the quantum phase transition that occurs at $\lambda_{c}=\frac{1}{2}$. Nevertheless, it is clear by inspection of Figs. 1 and 3 that results for the ground-state energy and also $M^{\text {trans. }}$ compare extremely well with the exact results of [44] for all values of $\lambda$, especially for the higher-order LSUB $n$ approximations with $n \gtrsim 6$. Although the results for $M^{z}$ in Fig. 2 also compare well, by inspection, with the exact results of [44] in the region where $M^{z}$ is known to be non-zero from these exact calculations, (i.e., $\lambda<\frac{1}{2}$.), the agreement is now much poorer outside this region (i.e., $\lambda>\frac{1}{2}$ ) for any of the LSUB $n$ approximants shown. However, even in this case, the agreement is found to become excellent when the LSUB $n$ sequence of approximants is extrapolated to the exact limit, $n \rightarrow \infty$, as alluded to in Sect. 2.1. Thus, a very well-tested extrapolation scheme for use in such cases where the system undergoes a quantum phase transition (see, e.g., [42] and references cited therein) is

$$
M^{z}(n)=\mu_{0}+\mu_{1} n^{-1 / 2}+\mu_{2} n^{-3 / 2},
$$

where $M^{z}(n)$ is the $n$ th-order CCM approximant (i.e., at the LSUB $n$ or SUB $n-n$ level) to $M^{z}$. Thus, in Fig. 2 we also show the extrapolation using Eq. (22) as the fitting formula, together with the LSUB $n$ approximants (for both the even values of $n$ shown and the unshown odd values) with $6 \leq n \leq 12$ as the input data, to determine the extrapolated value $\mu_{0}$, which is plotted. Clearly, the extrapolation now agrees extremely well with the exact result, even in the very sensitive region very close to the critical value $\lambda_{c}$, the value of which itself is now also predicted rather accurately.

\section{Spin-Half Triangular-Lattice Heisenberg Antiferromagnet in an External Magnetic Field}

We now consider the spin- $\frac{1}{2}$ triangular-lattice Heisenberg antiferromagnet in a magnetic field. The Hamiltonian that we will use here is given by

$$
\hat{H}=\sum_{\langle i, j\rangle} \hat{\mathbf{s}}_{i} \cdot \hat{\mathbf{s}}_{j}-\lambda \sum_{i=1}^{N} \hat{s}_{i}^{z},
$$

where the index $i$ runs over all $N$ lattice sites on the triangular lattice and the sum over the index $\langle i, j\rangle$ indicates a sum over all nearest-neighbour pairs, with each pair being counted once and once only. The strength of the applied external magnetic field is again given by $\lambda$. The triangular lattice is itself tripartite, being composed of three triangular sublattices, denoted as $A, B$ and $C$, the sites of which we denote respectively as $A_{n}, B_{n}$, and $C_{n}$. If the original lattice has a distance $a$ between nearest-neighbour sites, the corresponding distance on each of the sublattices $A, B$ and $C$ is $\sqrt{3} a$. 
It is easy to see that the classical spin- $S$ model corresponding to Eq. (23) (see, e.g., [45]) has an infinitely (and continuously) degenerate family of ground states, with the associated order parameter space being isomorphic to the 3D rotation group $\mathrm{SO}(3)$. Thus, one may readily rewrite the classical energy per spin for this model in the form

$$
\frac{E^{\mathrm{cl}}}{N}=\frac{1}{4 N} \sum_{k=1}^{2 N}\left(\mathbf{S}_{\Delta_{k}}-\frac{1}{3} \lambda\right)^{2}-\frac{3}{2} S^{2}-\frac{1}{18} \lambda^{2},
$$

where $\lambda=\lambda \hat{z}$ and $\hat{z}$ is a unit vector in the $z$-direction, and $\mathbf{S}_{\Delta_{k}} \equiv \mathbf{S}_{A_{\Delta_{k}}}+\mathbf{S}_{B_{\Delta_{k}}}+\mathbf{S}_{C_{\Delta_{k}}}$ is defined to be the sum of the three spins on the $k$ th elementary triangular plaquette on the lattice with nearest-neighbour vertices $A_{\Delta_{k}}, B_{\Delta_{k}}$, and $C_{\Delta_{k}}$. Equation (24) shows clearly that the energy is minimised when each of the squared terms in the sum over elementary triangular plaquettes is either zero (which is possible for $\lambda \leq 9 S$ ) or minimised [viz., to take the value $\left(3 S-\frac{1}{3} \lambda\right)^{2}$ for $\lambda>9 S$ ]. Thus, we find rather simply that the classical ground-state energy per spin is given by

$$
\frac{E_{g}^{\mathrm{cl}}}{N}= \begin{cases}-\frac{3}{2} S^{2}-\frac{1}{18} \lambda^{2} ; & \lambda \leq 9 S \\ 3 S^{2}-\lambda S ; & \lambda>9 S .\end{cases}
$$

For $\lambda \leq 9 S$, the ground state is clearly infinitely (and continuously) degenerate, since any configuration of spins that satisfies $\mathbf{S}_{\Delta_{k}}=\frac{1}{3} \lambda$ on all $2 N$ elementary triangular plaquettes will yield the same energy. Furthermore, this condition immediately yields that the comparable classical value for the lattice magnetisation $M$, where $\mathbf{M} \equiv \sum_{i=1}^{N} \mathbf{S}_{i}=M \hat{z}$ (i.e., in the direction of the field), in the ground state is given by

$$
\frac{M_{\mathrm{cl}}}{S}= \begin{cases}\frac{\lambda}{9 S} ; & \lambda \leq 9 S \\ 1 ; & \lambda>9 S\end{cases}
$$

from which we also see that the magnetisation saturates at the value $\lambda=\lambda_{s}=9 S$ of the magnetic field strength.

In the zero-field case $(\lambda=0)$ the energy-minimising condition (viz., that $\mathbf{S}_{\Delta_{k}}=0$ on all $2 N$ elementary triangular plaquettes) simply becomes the condition for the usual $120^{\circ}$ threesublattice Néel state. Associated with this state there is clearly a trivial degeneracy due to the rotational invariance of any Hamiltonian composed only of isotropic Heisenberg interactions, which is reflected in the ground-state order parameter space being isomorphic to the group $\mathrm{SO}(3)$. In the case of a finite external field $(\lambda \neq 0)$ the symmetry of the Hamiltonian of Eq. (23) is clearly reduced from $\mathrm{SO}(3)$ to $\mathrm{SO}(2) \times \mathrm{Z}_{3}$, corresponding to the rotational symmetry around the axis of the magnetic field and the discrete symmetry associated with the choice of the three sublattices $A, B$ and $C$. Despite this reduction in symmetry of the finite-field $(\lambda \neq 0)$ Hamiltonian of Eq. (23) from that of its zero-field $(\lambda=0)$ counterpart, the ground state of the former clearly shares the same [i.e., $\mathrm{SO}(3)$ ] degree of continuous degeneracy as that of the latter, due to the condition that $\mathbf{S}_{\Delta_{k}}=\frac{1}{3} \lambda$ on all $2 N$ elementary triangular plaquettes. Thus, on each plaquette, each of the three spins has two orientational degrees of freedom, and the above condition simply reduces the overall degrees of freedom from six to three. The trivial degeneracy of the $\lambda=0$ case is now, however, quite non-trivial in the $\lambda \neq 0$ case, since the local $120^{\circ}$ triangular-plaquette structures can become quite deformed by the application of the external field, even into non-coplanar configurations, as we now discuss.

From our discussion above, in principle, depending on the magnetic field strength, any of the five ground-state spin configurations sketched in Fig. 4 may appear. While the states I, II, III and IV are coplanar states, the "umbrella" state V is a 3D non-coplanar state. Although on the classical level both coplanar and non-coplanar sates are energetically degenerate, as we 

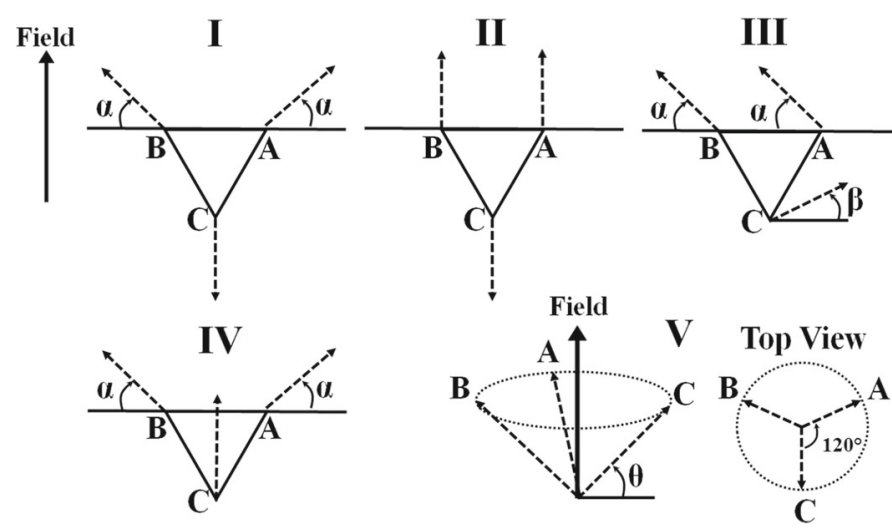

Fig. 4 Some examples of possible (degenerate) classical ground states (and hence also possible CCM model states) of the spin-half triangular-lattice Heisenberg antiferromagnet in an external magnetic field: states I-IV are coplanar, whereas state $\mathrm{V}$ is the non-coplanar (3D) "umbrella" state with spins at an angle $\theta$ to the plane perpendicular to the external field

have noted above for $\lambda \leq \lambda_{s}$, thermal fluctuations tend to favour the coplanar configurations [45-49].

By minimising the energy, it is easy to show that the classical spin- $S$ model described by Eq. (23) has a (coplanar) ground state of type I in Fig. 4 for $\lambda<3 S$, with a canting angle $\alpha$ given by

$$
\sin \alpha=\frac{1}{2}+\frac{\lambda}{6 S} ; \quad \lambda \leq 3 S .
$$

At zero field $(\lambda=0)$ state I simply becomes the usual $120^{\circ}$ three-sublattice Néel state, while precisely at the value $\lambda=3 S$ the state I becomes the collinear state II shown in Fig. 4, and as $\lambda$ is increased further the ground state now smoothly transforms into state III shown in Fig. 4. The canting angles $\alpha$ and $\beta$ are found to be given by

$$
\sin \alpha=\frac{\left(\lambda^{2}+27 S^{2}\right)}{12 \lambda S} ; \quad 3 S \leq \lambda \leq 9 S,
$$

and

$$
\sin \beta=\frac{\left(\lambda^{2}-27 S^{2}\right)}{6 \lambda S} ; \quad 3 S \leq \lambda \leq 9 S,
$$

which may readily be shown to satisfy the condition, $2 \cos \alpha=\cos \beta$, which ensures that state III does not acquire any lattice magnetisation transverse to the applied field. When the field strength takes the value $\lambda=3 S$ the angles are $\alpha=\frac{1}{2} \pi$ and $\beta=-\frac{1}{2} \pi$, which is again just equivalent to state II. As $\lambda$ is then increased, up to the saturation value $\lambda=\lambda_{s}=9 S$, the angle $\alpha$ first decreases to its minimum value, $\alpha=\frac{1}{3} \pi$, at $\lambda=3 \sqrt{3} S$, after which it again increases smoothly back to the value $\alpha=\frac{1}{2} \pi$ at $\lambda=9 S$. At the same time, as $\lambda$ is increased beyond the value $3 S$, the angle $\beta$ increases from $-\frac{1}{2} \pi$ to $\frac{1}{2} \pi$ at $\lambda=9 S$, taking the value $\beta=0$ in between, precisely at the point $\lambda=3 \sqrt{3} S$ where $\alpha$ becomes a minimum. For all values $\lambda>\lambda_{s}=9 S$ the ground state is the fully saturated ferromagnetic state (viz., state III with $\alpha=\frac{1}{2} \pi=\beta$ ). One may readily show that the classical ground-state energy of Eq. (23), for both states I and III at the respective values of their minimising canting angles and for the fully saturated state, is just that given previously in Eq. (25). Furthermore, the corresponding 
classical value for the lattice magnetisation (i.e., in the direction of the field) in the ground states I and III is given by our previous result of Eq. (26).

Although the state IV shown in Fig. 4 is not utilised as a CCM model state in any further application in this section to the spin- $\frac{1}{2}$ case of the Hamiltonian of Eq. (23), it will be considered later in Sect. 5. Hence, for completeness, we note that state IV has a minimum energy for the classical spin- $S$ case of the present Hamiltonian for a value of the canting angle $\alpha$ shown in Fig. 4 given by

$$
\sin \alpha=-\frac{1}{2}+\frac{\lambda}{6 S} ; \quad \lambda \leq 9 S
$$

Hence, unlike the situation for state I, which undergoes a smooth transformation to state III at a value, $\lambda=\frac{1}{3} \lambda_{s}$, of the external field strength, (which then itself smoothly varies as $\lambda$ is further increased up to the value $\lambda_{s}$, at which point it becomes the fully saturated ferromagnetic state), state IV simply varies smoothly from the $120^{\circ}$ three-sublattice Néel state at zero field, $\lambda=0$, to the fully saturated ferromagnetic state at $\lambda=\lambda_{s}$.

For the classical case, as we have already noted, a non-coplanar state of the form of state $\mathrm{V}$ of Fig. 4 is degenerate in energy with states I and III above in their respective regimes. Thus, one readily finds that state $\mathrm{V}$ has a minimum energy for the classical spin- $S$ Hamiltonian of Eq. (23) for a value of the out-of-plane angle $\theta$ given by

$$
\sin \theta= \begin{cases}\frac{\lambda}{9 S} ; & \lambda \leq 9 S \\ 1 ; & \lambda>9 S\end{cases}
$$

Thus, with that value of $\theta$, state $\mathrm{V}$ also yields a value for the energy identical to that of Eq. (25). Clearly, the lattice magnetisation is then also given by Eq. (26).

For the quantum spin- $\frac{1}{2}$ case, no exact solution is available, but many investigations $[30,36,46,50-66]$ have demonstrated that the order from disorder mechanism [67,68] selects coplanar spin configurations, and, in particular, a wide magnetisation plateau at one-third of the saturation value (i.e., at $\lambda=\frac{1}{3} \lambda_{s}$ ) is present $[30,36,46,50-66]$. This is precisely the value of the field strength for which the collinear state II is degenerate with other ground-state spin configurations. Since it is well known that quantum fluctuations tend to favour collinear over non-collinear spin configurations, it is no real surprise that in the extreme quantum limiting case $S=\frac{1}{2}$ the classical transition point at $\lambda=\frac{1}{3} \lambda_{s}$ should broaden into a plateau. A previous investigation using the CCM [30] with the coplanar states I, II and III as the model states showed that for the spin- $\frac{1}{2}$ model the plateau state occurs for $1.37 \lesssim \lambda \lesssim 2.15$. Here our aim is to compare new CCM results generated with the 3D "umbrella" state V as the model state to those obtained previously for the coplanar states [30].

According to the CCM scheme briefly outlined in Sect. 2 we have to perform a passive rotation of the local spin axes of the spins such that all spins appear to point downwards for all five model states I, II, III, IV and V in Fig. 4. For the coplanar model states I, II, and III that procedure has been explained and discussed in detail in [30]. A similar rotation is also necessary for the coplanar model state IV, that does not play a role in this section, but which will be used in Sect. 5. For the non-coplanar model state V, that comprises spins that make an angle $\theta$ to the plane perpendicular to the external field, the derivation of the Hamiltonian after rotation of the local spin axes is given in Appendix B, from which we note that the final result is given by 


$$
\begin{aligned}
\hat{H}= & \sum_{\left\langle i_{B, C, A} \rightarrow j_{C, A, B}\right\rangle}\left\{\left(\sin ^{2} \theta-\frac{1}{2} \cos ^{2} \theta\right) \hat{s}_{i_{B, C, A}}^{z} \hat{s}_{j_{C, A, B}}^{z}\right. \\
& +\frac{1}{4}\left(\frac{1}{2} \sin ^{2} \theta-\cos ^{2} \theta-\frac{1}{2}\right)\left(\hat{s}_{i_{B, C, A}}^{+} \hat{s}_{j_{C, A, B}}^{+}+\hat{s}_{i_{B, C, A}}^{-} \hat{s}_{j_{C, A, B}}^{-}\right) \\
& +\frac{1}{4}\left(\cos ^{2} \theta-\frac{1}{2} \sin ^{2} \theta-\frac{1}{2}\right)\left(\hat{s}_{i_{B, C, A}}^{+} \hat{s}_{j_{C, A, B}}^{-}+\hat{s}_{i_{B, C, A}}^{-} \hat{s}_{j_{C, A, B}}^{+}\right) \\
& \left.+\frac{\sqrt{3}}{4} \cos \theta\left[\hat{s}_{i_{B, C, A}}^{z}\left(\hat{s}_{j_{C, A, B}}^{+}+\hat{s}_{j_{C, A, B}}^{-}\right)-\left(\hat{s}_{i_{B, C, A}}^{+}+\hat{s}_{i_{B, C, A}}^{-}\right) \hat{s}_{j_{C, A, B}}^{z}\right]\right\} \\
& +\lambda \sum_{i=1}^{N} \sin \theta \hat{s}_{i}^{z} \\
& +\mathrm{i} \sum_{\left\langle i_{B, C, A} \rightarrow j_{C, A, B}\right\rangle}\left\{\frac{\sqrt{3}}{4} \sin \theta\left(\hat{s}_{i_{B, C, A}}^{-} \hat{s}_{j_{C, A, B}}^{+}-\hat{s}_{i_{B, C, A}}^{+} \hat{s}_{j_{C, A, B}}^{-}\right)\right. \\
& \left.+\frac{3}{4} \sin \theta \cos \theta\left[\hat{s}_{i_{B, C, A}}^{z}\left(\hat{s}_{j_{C, A, B}}^{+}-\hat{s}_{j_{C, A, B}}^{-}\right)+\left(\hat{s}_{i_{B, C, A}}^{+}-\hat{s}_{i_{B, C, A}}^{-}\right) \hat{s}_{j_{C, A, B}}^{z}\right]\right\} \\
& +\mathrm{i} \frac{\lambda}{2} \sum_{i=1}^{N} \cos \theta\left(\hat{s}_{i}^{+}-\hat{s}_{i}^{-}\right),
\end{aligned}
$$

where the sums over $\left\langle i_{B, C, A} \rightarrow j_{C, A, B}\right\rangle$ represent a shorthand notation to include the three sorts of "directed" nearest-neighbour bonds on each basic triangular plaquette of side $a$ on the triangular lattice, which join sites $i_{B}$ and $j_{C}$ going from the $B$-sublattice to the $C$-sublattice, sites $i_{C}$ and $j_{A}$ going from the $C$-sublattice to the $A$-sublattice, and sites $i_{A}$ and $j_{B}$ going from the $A$-sublattice to the $B$-sublattice (in those directions only and not reversed). We see that this Hamiltonian now contains terms with both real and imaginary coefficients.

Clearly, when using any classical configuration of spins as a CCM model state, such as those shown in Fig. 4, there is no reason to expect that the quantum spin- $S$ version of the model, with a finite value of the spin quantum number $S$, will take the same values of the angle parameters that characterise it as the classical version (i.e., in the $S \rightarrow \infty$ limit), even in the case that the quantum ground state (at least partially) preserves the classical ordering inherent in the model state. For this reason a first step in using any such model state in a CCM calculation is to optimise the angle parameters that characterise the spin configuration. To do so we simply choose those parameters that minimise the ground-state energy at each (either $\mathrm{LSUB} n$ or $\mathrm{SUB} n-n)$ level of approximation that we undertake, performing a separate such optimisation at each level.

Typical such CCM results for the ground-state energy of the spin- $\frac{1}{2}$ Hamiltonian described by Eq. (23) from using the non-coplanar state $\mathrm{V}$ as the model state are shown in Fig. 5 as a function of the out-of-plane angle $\theta$ and for various values of the external field strength in the range $0 \leq \lambda \leq \lambda_{s}$, for the particular case of the LSUB5 level of approximation. The groundstate energy is found to be a real number for all values of $\lambda$ and $\theta$. We note in particular that all purely imaginary contributions to the energy sum identically to zero. Figure 5 demonstrates the general result that the ground-state energy has a well-defined minimum with respect to the angle for all values of $\lambda$, at each LSUB $n$ level of approximation. The angle that minimises the ground-state energy is plotted as a function of $\lambda$ in Fig. 6 for various levels of LSUB $n$ approximation. As required, this angle is zero (i.e., the model state is coplanar) when the external field is zero $(\lambda=0)$. Also as required, all spins point in the direction of the field (i.e., 


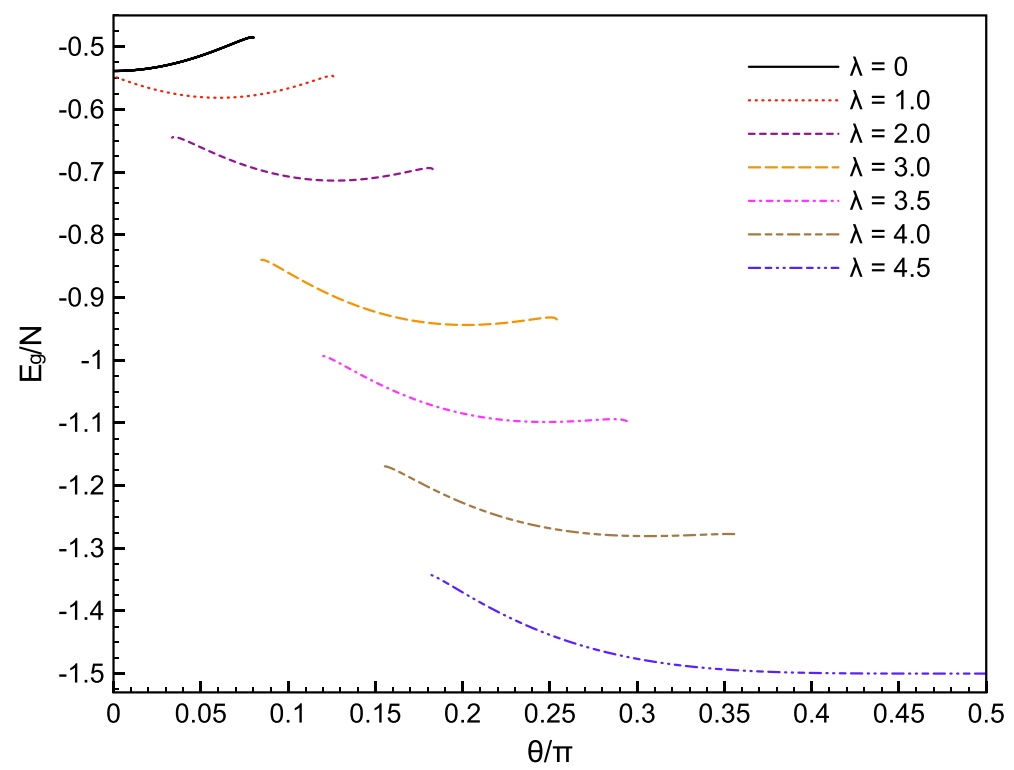

Fig. $5 \mathrm{CCM}$ results for the ground-state energy per site, $E_{g} / N$, of the spin-half triangular-lattice Heisenberg antiferromagnet, calculated at the LSUB5 level of approximation, plotted as a function of the out-of-plane angle $\theta$ (in units of $\pi$ ) for the 3D non-coplanar state $\mathrm{V}$, shown for various values of the external magnetic field strength, $\lambda$ in the range between zero and the saturation value $\lambda_{s}=\frac{9}{2}$

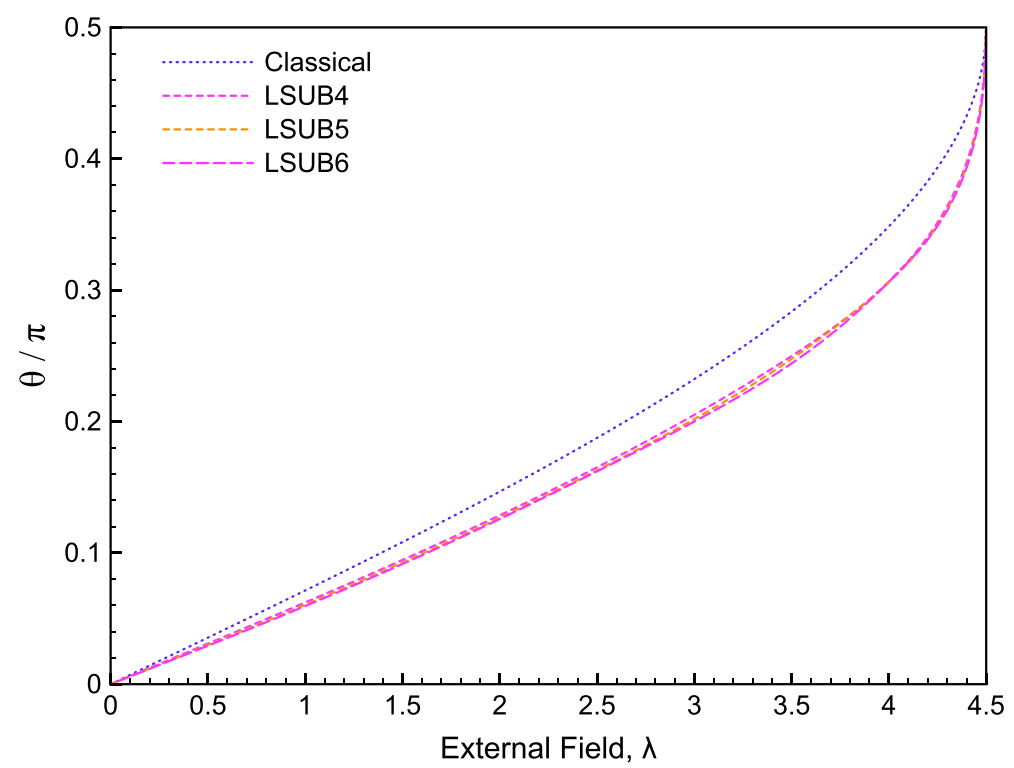

Fig. $6 \mathrm{CCM}$ results for the out-of-plane angle $\theta$ (in units of $\pi$ ) that minimises the ground-state energy of the spin-half triangular-lattice Heisenberg antiferromagnet in an external magnetic field of strength $\lambda$, plotted as a function of $\lambda$ for the 3D non-coplanar state V, at various LSUBn levels of approximation. For comparison purposes we also show the corresponding classical result from Eq. (31) with $S=\frac{1}{2}$ 


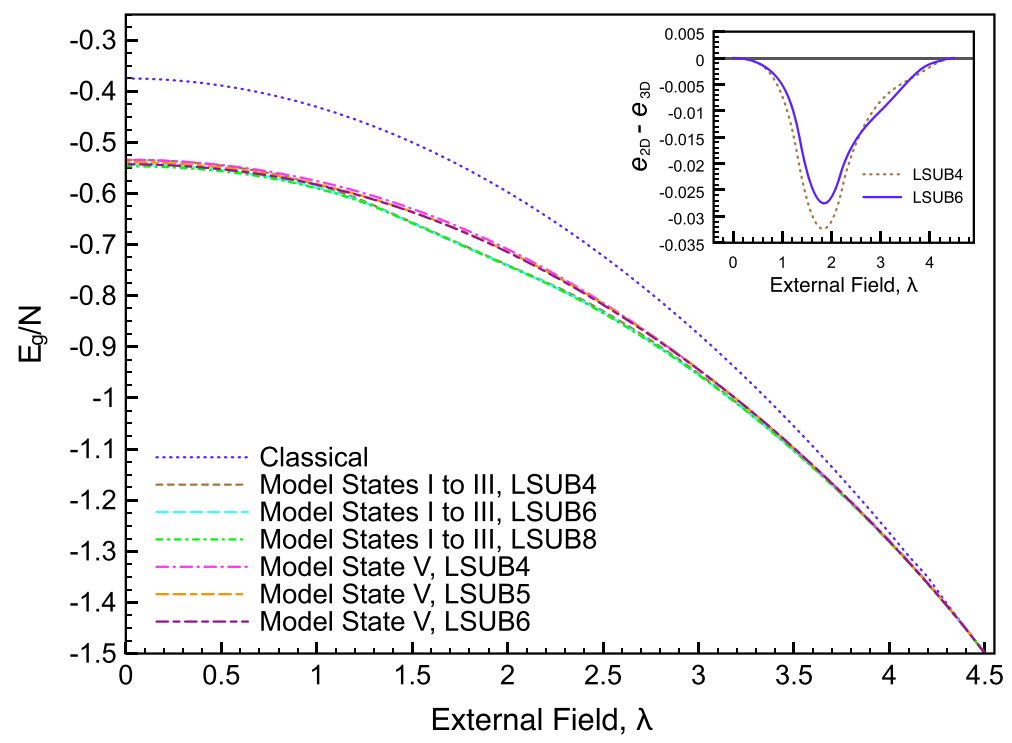

Fig. 7 Main: CCM results for the ground-state energy per site, $E_{g} / N$, of the spin-half triangular-lattice Heisenberg antiferromagnet in an external magnetic field of strength $\lambda$, plotted as a function of $\lambda$, using both coplanar states I-III (results from [30]) and the 3D non-coplanar state V as CCM model states, at various LSUB $n$ levels of approximation. For comparison purposes we also show the corresponding classical result from Eq. (25) with $S=\frac{1}{2}$. Inset: Energy difference, $\delta e=e_{2 \mathrm{D}}-e_{3 \mathrm{D}}$ (where $e \equiv E_{g} / N$ ), between the 2D coplanar states and the 3D non-coplanar state

shown by $\theta / \pi=\frac{1}{2}$ ) at the saturation field, $\lambda_{s}=\frac{9}{2}$. The angle that minimises the energy varies continuously as a function of $\lambda$ for model state V, excepting the limiting point at "saturation", $\lambda_{s}$. Furthermore, we see from Fig. 6 that the non-coplanar energy-minimising configuration of spins converges very rapidly as the LSUB $n$ approximation index $n$ is increased, for all values of $\lambda$.

Ground-state energies for model state $\mathrm{V}$ are shown in Fig. 7, in which results for the coplanar model states I-III from [30] are also shown for comparison. Again, LSUB $n$ results for the energy converge rapidly with increasing levels of the truncation index $n$ for all values of $\lambda$. We see very clearly that ground-state energies for the coplanar model states lie lower than those of the 3D "umbrella" state (model state V) for all values $\lambda$, which is in agreement with the results of other methods [45-47]. Note that the energy difference between the coplanar and the non-coplanar states is particularly large in the plateau region around $\lambda=1.5$, as can be seen clearly from the inset in Fig. 7.

Naturally, the (physical) lattice magnetisation is defined in terms of the spin directions before all rotations of the local spin axes have been carried out. Thus, the lattice magnetisation is given in terms of the "unrotated" coordinates as:

$$
M=\frac{1}{N} \sum_{i=1}^{N}\left\langle\tilde{\Psi}\left|\hat{s}_{i}^{z}\right| \Psi\right\rangle
$$

After the rotations of the local spin axes for model state V, which led to the expression of Eq. (32), have been completed, this expression is given by 


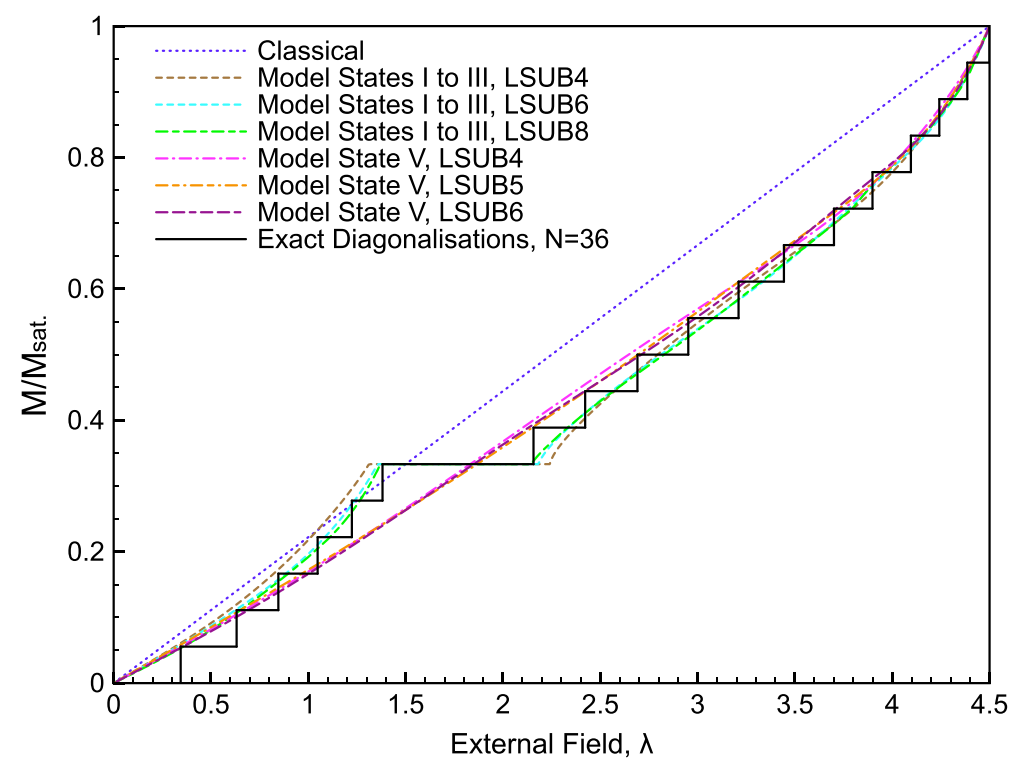

Fig. $8 \mathrm{CCM}$ results at various levels of LSUB $n$ approximation for the ratio, $M / M_{\mathrm{sat}}$, of the lattice magnetisation to its saturated value, of the spin-half triangular-lattice Heisenberg antiferromagnet in an external magnetic field of strength $\lambda$, plotted as a function of $\lambda$, using both coplanar states I-III (results from [30]) and the 3D non-coplanar model state V as CCM model states. For comparison purposes we also show the corresponding classical result from Eq. (26), as well as the result from an exact diagonalisation of the Hamiltonian on a (36-site) finite-sized lattice

$$
M=-\frac{1}{N} \sum_{i=1}^{N}\left\langle\tilde{\Psi}\left|\left(\sin \theta \hat{s}_{i}^{z}+\frac{\mathrm{i}}{2} \cos \theta\left[\hat{s}_{i}^{+}-\hat{s}_{i}^{-}\right]\right)\right| \Psi\right\rangle .
$$

Previous initial results for model state V [31] used computational differentiation and the Hellmann-Feynman theorem to evaluate this lattice magnetisation of Eq. (33). Here we evaluate it directly by finding both the ket- and bra-state correlation coefficients, which are now complex-valued, and then evaluating the expectation value explicitly, although we note that this method provides identical results (within the precision allowed by numerical differentiation) to that of the former technique, as required.

Once again, the results for the lattice magnetisation shown in Fig. 8 for model state V are found to be real numbers for all values of $\lambda$, with all imaginary contributions summing identically to zero. Furthermore, $\mathrm{LSUB} n$ results are again found to converge with increasing approximation level $n$ for all values of $\lambda$. It is evident that CCM results for the $3 \mathrm{D}$ "umbrella" model state $\mathrm{V}$ do not indicate the presence of the well-known magnetisation plateau that occurs in this system. By contrast, results for the coplanar states agree well with those results of exact diagonalisations, including the well-known plateau regime at $M / M_{\text {sat. }}=1 / 3$. The high-order LSUB8 approximation for the coplanar states, for example, yields [30] that this regime extends over the region $1.37 \lesssim \lambda \lesssim 2.15$, and it is clear too from Fig. 8 that the borders of the plateau region also converge rapidly as the order $n$ of the LSUB $n$ approximation is increased. Such CCM results [30] for the plateau for the coplanar states that we have now shown explicitly lie lower in energy than the 3D "umbrella" state, are in excellent agreement with experimental results for the magnetic compound $\mathrm{Ba}_{3} \mathrm{CoSb}_{2} \mathrm{O}_{9}$ (a spin-half triangular-lattice antiferromagnet) and exact diagonalisations [69]. Note also that previous 
CCM results for the coplanar model states also indicate that a similar plateau occurs over the range $2.82 \lesssim \lambda \lesssim 3.70$ for the for the spin-one triangular-lattice antiferromagnet, and this theoretical result has subsequently been established experimentally for the compound $\mathrm{Ba}_{3} \mathrm{NiSb}_{2} \mathrm{O}_{9}$ (a spin-one triangular-lattice antiferromagnet) [36].

\section{Spin-S Triangular-Lattice XXZ Antiferromagnet in an External Magnetic Field}

In recent investigations [70-75] of the anisotropic triangular-lattice $X X Z$ model

$$
\hat{H}=\sum_{\langle i, j\rangle}\left(\hat{s}_{i}^{x} \hat{s}_{j}^{x}+\hat{s}_{i}^{y} \hat{s}_{j}^{y}+\Delta \hat{s}_{i}^{z} \hat{s}_{j}^{z}\right)-\lambda \sum_{i=1}^{N} \hat{s}_{i}^{z},
$$

where the indices have the same meaning as in Eq. (23), it has been shown that for an easyplane anisotropy (i.e., $\Delta<1$ ) the 3D "umbrella" state V discussed in Sect. 4 can become energetically favoured over the coplanar states, so as to form the true ground state under certain conditions that we now elaborate.

The corresponding phase diagram in the $\Delta-\lambda$ plane is rich, (see, e.g., [70,71]). Moreover, the phase boundary between the coplanar and non-coplanar ground states strongly depends on the spin quantum number $S$. We note that in the classical limit $S \rightarrow \infty$ for $\Delta<1$ the non-coplanar "umbrella" state is always energetically favoured over the planar states to form the ground state, as we elaborate further below, whereas for the extreme quantum case $S=\frac{1}{2}$ there is a wide region of values of the anisotropy parameter $\Delta$ and the field strength $\lambda$ where coplanar states are favoured. We note further that since the energy differences between competing ground states can be very small, accurate and self-consistent calculations are hence required to be able to distinguish between them reliably.

By comparison with the derivation of Eq. (24) for the Hamiltonian of Eq. (23) in Sect. 4, it is clear that we may write the classical energy per spin for the current anisotropic triangularlattice $X X Z$ model in the form

$$
\frac{E^{\mathrm{cl}}}{N}=\frac{1}{4 N} \sum_{k=1}^{2 N}\left(\mathbf{S}_{\Delta_{k}}-\frac{1}{3} \lambda\right)^{2}+(\Delta-1) \sum_{\langle i, j\rangle} S_{i}^{z} S_{j}^{z}-\frac{3}{2} S^{2}-\frac{1}{18} \lambda^{2} .
$$

It is evident that the second sum in Eq. (36) can now potentially favour non-coplanar states in the case of easy-plane anisotropy (i.e., when $\Delta<1$ ). One readily finds, by making use of Eq. (36), that state $\mathrm{V}$ of the form shown in Fig. 4 has a minimum energy for the classical spin- $S$ Hamiltonian of Eq. (35) for a value of the out-of-plane angle $\theta$ given by

$$
\sin \theta= \begin{cases}\frac{\lambda}{\lambda_{s}} ; & \lambda \leq \lambda_{s} \\ 1 ; & \lambda>\lambda_{s}\end{cases}
$$

where $\lambda_{s} \equiv 3(1+2 \Delta) S$ is the value of the field strength that reaches saturation (i.e., the fully aligned ferromagnetic state) for this state $\mathrm{V}$. With this value of $\theta$ one may readily show that state $\mathrm{V}$ yields a value for the classical ground-state energy per spin given by

$$
\frac{E_{g}^{\mathrm{cl} ; \mathrm{V}}}{N}= \begin{cases}-\frac{3}{2} S^{2}-\frac{\lambda^{2}}{6(2 \Delta+1)} ; & \lambda \leq \lambda_{s} \\ 3 \Delta S^{2}-\lambda S ; & \lambda>\lambda_{s},\end{cases}
$$


which also replicates Eq. (25) at isotropy (i.e., when $\Delta=1$ ). Furthermore, one may readily show that state $\mathrm{V}$, with the energy-minimising value of the out-of-plane angle $\theta$ of Eq. (37), yields a classical value for the lattice magnetisation given by

$$
\frac{M_{\mathrm{cl} ; \mathrm{V}}}{S}= \begin{cases}\frac{\lambda}{\lambda_{s}} ; & \lambda \leq \lambda_{s} \\ 1 ; & \lambda>\lambda_{s}\end{cases}
$$

We may compare the above results for the non-coplanar state $\mathrm{V}$ with those of the coplanar states. For example, one may readily show, again by making use of Eq. (36), that state IV of the form shown in Fig. 4 has a minimum energy for the classical spin- $S$ Hamiltonian of Eq. (35) for a value of the canting angle $\alpha$ given by

$$
\sin \alpha= \begin{cases}\frac{-\Delta+\lambda /(3 S)}{(1+\Delta)} ; & \lambda \leq \lambda_{s} \\ 1 ; & \lambda>\lambda_{s}\end{cases}
$$

where $\lambda_{s}=3(1+2 \Delta) S$ as before. Once again, this result is in accord with our previous result of Eq. (30) at the isotropic point, $\Delta=1$. With this value of $\alpha$ one may show that state IV yields a value for the classical ground-state energy per spin given by

$$
\frac{E_{g}^{\mathrm{cl} ; \mathrm{IV}}}{N}= \begin{cases}\frac{1}{(\Delta+1)}\left[-\left(\Delta^{2}+\Delta+1\right) S^{2}+\frac{1}{3}(\Delta-1) \lambda S-\frac{1}{9} \lambda^{2}\right] ; & \lambda \leq \lambda_{S} \\ 3 \Delta S^{2}-\lambda S ; & \lambda>\lambda_{s},\end{cases}
$$

which also replicates Eq. (25) at isotropy (i.e., when $\Delta=1$ ). One may also show that state $\mathrm{IV}$, with the energy-minimising value of the canting angle $\alpha$ of Eq. (40) yields a classical value for the lattice magnetisation given by

$$
\frac{M_{\mathrm{cl} ; \mathrm{IV}}}{S}= \begin{cases}\frac{\left(9 S+4 \lambda-\lambda_{s}\right)}{3\left(3 S+\lambda_{s}\right)} ; & \lambda \leq \lambda_{s} \\ 1 ; & \lambda>\lambda_{s}\end{cases}
$$

which may be compared with the corresponding result of Eq. (39) for state V.

Finally, at the classical level, one may readily show from Eqs. (38) and (41) that the difference between the minimum energy per spin for the "umbrella" state $\mathrm{V}$ and that for the coplanar state IV is given explicitly by

$$
\frac{E_{g}^{\mathrm{cl} ; \mathrm{IV}}}{N}-\frac{E_{g}^{\mathrm{cl} ; \mathrm{V}}}{N}=\frac{(1-\Delta)}{18(\Delta+1)(2 \Delta+1)}\left(\lambda-\lambda_{s}\right)^{2} ; \quad \lambda \leq \lambda_{s} .
$$

It is evident from Eq. (43) that the "umbrella' state V always lies lower in energy than the coplanar state IV for all values of the anisotropy parameter $\Delta<1$, as we have already asserted, in the classical limit $S \rightarrow \infty$. Equation (43) shows clearly, however, that the energy difference between the states decreases both as $\lambda$ approaches the saturation value $\lambda_{s}$ and as $\Delta$ approaches unity (i.e., near the Heisenberg isotropic limit). One expects on rather general grounds that quantum fluctuations will favour phases with coplanar over noncoplanar configurations of spins. One also expects that the effects of quantum fluctuations will increase monotonically as the spin quantum number $S$ is decreased smoothly from the large- $S$ classical limit. Hence, for small positive values of $\left(\lambda_{s}-\lambda\right)$ it is, a priori, likely that the value $\Delta_{1}(S)$ of the anisotropy parameter at which a possible quantum phase transition occurs between the "umbrella" state forming the ground state (for $\Delta<\Delta_{1}$ ) to a coplanar state forming the ground state (for $\Delta>\Delta_{1}$ ) would decrease smoothly as $S$ decreases from the classical value $\Delta_{1}(\infty)=1$ at $S \rightarrow \infty$ towards a lower value $\Delta\left(\frac{1}{2}\right)$ at the extreme quantum limit, $S=\frac{1}{2}$. 
Thus, to demonstrate the capability of the new 3D CCM code to detect such anisotropydriven quantum transitions between coplanar and non-coplanar states we now consider the same model described by the Hamiltonian of Eq. (35) for values of the field strength near to the saturation value, $\lambda_{s}=3(1+2 \Delta) S$, but for various finite values of the spin quantum number, viz., $S=\frac{1}{2}, 1, \ldots, 5$. The local spin rotations are identical to those discussed in Sect. 4 and Appendix B for model states I, II, III, and V, and are hence not repeated here. Model state IV uses the same rotations as for model state I for the $A$ and $B$ sublattices, although the "up" spins on the $C$ sublattice for this model state obviously also require an additional rotation of $180^{\circ}$.

Other investigations [70-75] have shown that for strong magnetic fields with a strength $\lambda$ infinitesimally below the saturation field strength $\lambda_{s}$ the states III, IV or V shown in Fig. 4 appear as the ground state, depending on the value of the anisotropy parameter $\Delta$. In the $X Y$ limit, $\Delta=0$, the $3 \mathrm{D}$ "umbrella" state $\mathrm{V}$ is always the ground state, for all values of the spin quantum number $S$. Increasing $\Delta$ then leads first to a transition to the coplanar state IV at a critical value $\Delta_{1}(S)$ and then to a second transition at $\Delta_{2}(S)$ to the coplanar state III. Both transition points depend on the value of $S$. To find the transition points with our $\mathrm{CCM}$ approach is straightforward but computationally quite intensive, since for each spin quantum number $S$ the energies of the competing ground states have to be computed for a fine net of $\Delta$ values, for each of which the corresponding quantum pitch angles that minimise the respective energies at the particular $\mathrm{SUB} n-n$ level of approximation being utilised must be determined iteratively. Moreover, the size of the set of coupled nonlinear CCM ket-state equations increases rapidly with the truncation index $n$, such that for the highest SUB6-6 level of approximation considered here the number of such equations is $N_{f}(6)=80,339$. Therefore, we focus particular attention here on the transition between the 3D "umbrella" state $\mathrm{V}$ and the coplanar state IV, which we have already discussed above in the classical limit, $S \rightarrow \infty$.

In Fig. 9 we show two examples of the energy difference $\delta e=e_{\mathrm{IV}}-e_{\mathrm{V}}$ between the two competing states IV and $\mathrm{V}$, where $e \equiv E / N$ represents the energy per spin in each case. The change of the sign of $\delta e$, as $\Delta$ is varied across the critical value $\Delta_{1}$, is obvious. Note that a change of the sign of $\delta e$ with decreasing $\lambda$ at fixed $\Delta$ (see, e.g., the green line for $\Delta=0.86$ in the lower panel of Fig. 9), does not actually indicate a reentrance of the "umbrella" state $\mathrm{V}$, since for decreasing $\lambda$ the coplanar state III, which we have not considered here, actually becomes the ground state (see, e.g., [70-72,74,75]). Based on curves such as those shown in Fig. 9 we derive the critical points $\Delta_{1}(S)$ with an accuracy of \pm 0.015 , as shown in Fig. 10 . We compare our CCM data with the large- $S$ approach of Starykh et al. [72] which yields $\Delta_{1}(S)=1-0.53 / S$, as well as with numerical data obtained by the dilute Bose gas expansion [74]. As can be clearly seen, there is good agreement of our results with both those of [74] and those of [72], except where the latter results from the large- $S$ expansion naturally fail for the smaller values of $S$. Finally, we note too that our results also agree extremely well with those from a recent study that used the numerical cluster mean-field plus scaling method [75] to investigate the cases with $S \leq \frac{3}{2}$. To conclude, we have shown that the new CCM code for $3 \mathrm{D}$ non-coplanar ground states provides accurate data that, in particular, allow us to examine with confidence the quantum selection of competing ground states of the frustrated triangular-lattice $X X Z$ antiferromagnet in an external magnetic field. 

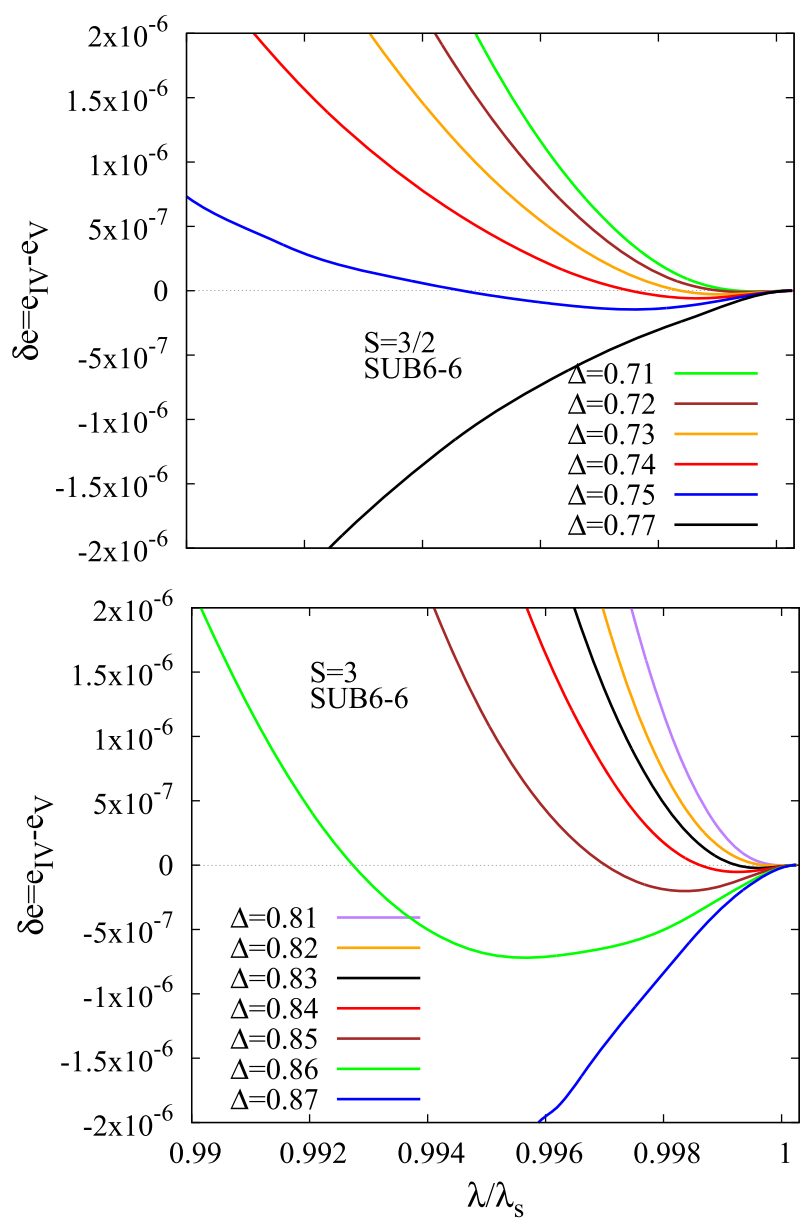

Fig. 9 CCM SUB6-6 results for the difference of energies per spin, $\delta e=e_{\mathrm{IV}}-e_{\mathrm{V}}$, between the two competing states IV and V, of a spin- $S$ anisotropic triangular-lattice $X X Z$ antiferromagnet in an external magnetic field of strength $\lambda$, plotted as a function of $\lambda$ (in units of $\lambda_{s}$ ), for values of $\lambda$ just below the saturation field strength, $\lambda_{S}=3 S(1+2 \Delta)$, for various values of the anisotropy parameter, $\Delta$, and for two values of the spin quantum number, $S=3 / 2$ (top panel) and $S=3$ (bottom panel)

\section{Summary}

Coplanar model states for applications of the CCM to problems in quantum magnetism are those states in which all spins lie in a plane, whereas 3D model states are non-coplanar states in which the spins do not lie in any plane. The first step in applying the CCM to lattice quantum spin systems is always to rotate the local spin axes (i.e., on each lattice site) so that all spins in our model state appear mathematically to points in the downwards (i.e., negative) $z$-direction. We have shown explicitly here that this process leads inevitably to terms in the rotated Hamiltonian with complex-valued coefficients for 3D model states, by contrast with the case for coplanar model states, where all of the respective terms carry (or can be made to carry) real-valued coefficients. Since these rotations represent unitary transformations the rotated Hamiltonians in each case are still Hermitian. Nevertheless, for the case of 3D model 


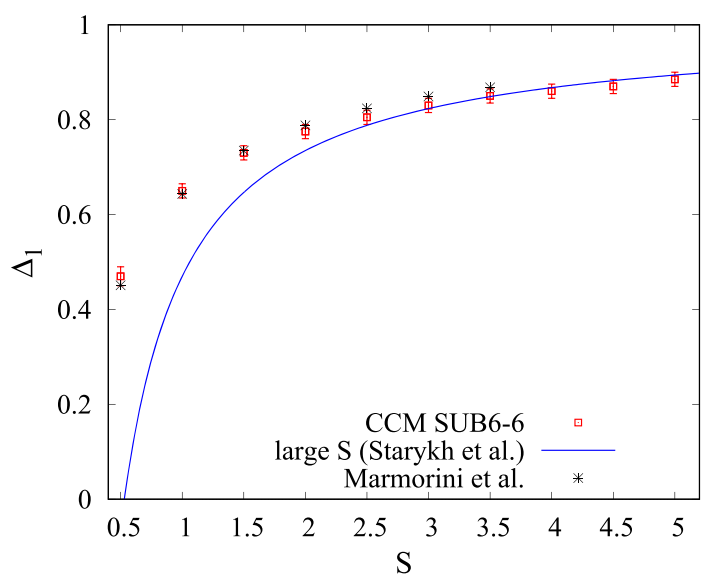

Fig. 10 CCM SUB6-6 results with error bars as shown for the critical value $\Delta_{1}$ of the anisotropy parameter $\Delta$, which denotes the point at which the stable ground state changes from being state $\mathrm{V}$ (for $\Delta<\Delta_{1}$ ) to state IV (for $\Delta>\Delta_{1}$ ), of a spin- $S$ anisotropic triangular-lattice $X X Z$ antiferromagnet in an external magnetic field of strength infinitesimally below the saturation field strength, $\lambda_{s}=3 S(1+2 \Delta)$, versus the spin quantum number $S$. The (blue) solid line corresponds to the large- $S$ expansion result, $\Delta_{1}=1-0.53 / S$, of [72]. The (black) stars correspond to the dilute Bose gas expansion results of [74]

states, even though the expectation values of all physical operators are hence guaranteed to be real-valued quantities, the intervening CCM bra- and ket-state multispin cluster coefficients from which they will be calculated will be complex-valued at all levels of approximation.

In this paper we have demonstrated that the existing high-order CCM code [43] can be extended appropriately to be able to be applied to such rotated Hamiltonians with complexvalued coefficients that arise from the utilisation of 3D model states. An explicit derivation of such a Hamiltonian after all rotations of spin axes for a 3D model state was given for the triangular-lattice Heisenberg antiferromagnet in an external magnetic field. Although in such cases the CCM ket- and bra-state equations for the multispin cluster correlation coefficients are now complex-valued quantities, we have demonstrated explicitly for several models of interest that all expectation values are real numbers. This was also shown explicitly in analytical LSUB1 calculations for the one-dimensional spin- $\frac{1}{2}$ Ising ferromagnet in a transverse external magnetic field.

Due to the length and complexity of the CCM code, its extension from coplanar to 3D model states is a non-trivial task. Furthermore, the task of defining the problem to be solved by the code in CCM "script files" becomes more difficult. Hence, this is an important advance for practical applications of the CCM, and one that greatly extends its range of applicability.

Finally, excellent correspondence with the results of other methods was found for all of the cases considered here, namely, (a) the $1 \mathrm{D}$ spin- $\frac{1}{2}$ Ising ferromagnetic chain in a transverse external magnetic field; (b) the 2D spin- $\frac{1}{2}$ Heisenberg antiferromagnet on a triangular lattice in the presence of an external magnetic field; and (c) the $2 \mathrm{D}$ spin- $S$ triangular-lattice $X X Z$ antiferromagnet in the presence of an external magnetic field, for the cases $\frac{1}{2} \leq S \leq 5$. For the first case of the transverse Ising model, which simply provides a testbed for which we can artificially introduce terms in a rotated Hamiltonian pertaining to it that contain an imaginary coefficient, we showed that CCM results agree well with exact results. With the extended CCM methodology thereby validated we turned to two frustrated 2D models defined on a triangular lattice, for both of which a real 3D non-coplanar configuration of spins is physically relevant. 
Our CCM results for the spin- $\frac{1}{2}$ triangular-lattice Heisenberg antiferromagnet show that coplanar ordering is favoured over non-coplanar ordering for all values of the applied external magnetic field, which agrees with the results of other approximate methods [45-47]. The boundary between a 3D ground state (state V) and a coplanar ground state (state IV) was also obtained for the $X X Z$ model on the triangular lattice for values of the external magnetic field near to saturation and for spin quantum number $S \leq 5$. The CCM calculations in this case were computationally intensive for this frustrated model, especially for high spin quantum numbers. However, we note that only a very few other approximate methods can deal effectively and accurately with the combination of strong frustration and higher spin quantum number. The differences in ground-state energies between the two states is also very small in the limit of field saturation. Hence, an accurate delineation of the phase boundary is an extremely delicate task. Despite this inherent difficulty excellent correspondence was seen with the results of other approximate methods. These results thereby constitute a useful advance in the understanding of this model, as well as providing an excellent quantitative test of high-order CCM using 3D model states.

Open Access This article is distributed under the terms of the Creative Commons Attribution 4.0 International License (http://creativecommons.org/licenses/by/4.0/), which permits unrestricted use, distribution, and reproduction in any medium, provided you give appropriate credit to the original author(s) and the source, provide a link to the Creative Commons license, and indicate if changes were made.

\section{Appendix A: LSUB1 Calculation for the 1D Spin- $\frac{1}{2}$ Ising Ferromagnet in a Transverse Magnetic Field}

We first carry out a CCM LSUB1 calculation for the spin- $\frac{1}{2} 1 \mathrm{D}$ transverse Ising model of Eq. (13). We recall that the CCM model state contains spins that point in the downward $z$-direction. We note also that this is the exact (albeit trivial) ground state when $\lambda=0$ and that all CCM correlation coefficients should therefore tend to zero in this limit, $\lambda \rightarrow 0$. The LSUB1 ket-state operator $\bar{S}$ is thus given by

$$
\hat{S}=a \sum_{i=1}^{N} \hat{s}_{i}^{+} .
$$

By making use of the usual SU(2) spin commutation relations, $\left[\hat{s}_{l}^{+}, \hat{s}_{l^{\prime}}^{-}\right]=2 \hat{s}_{l}^{z} \delta_{l, l^{\prime}}$ and $\left[\hat{s}_{l}^{z}, \hat{s}_{l^{\prime}}^{ \pm}\right]= \pm \hat{s}_{l}^{ \pm} \delta_{l, l^{\prime}}$, and by also using the nested commutator expansion of Eq. (10), it is readily proven that the CCM similarity transforms of the operators $\hat{s}_{l}^{+}, \hat{s}_{l}^{z}$, and $\hat{s}_{l}^{-}$are given by

$$
\begin{aligned}
\mathrm{e}^{-\hat{S}} \hat{s}_{l}^{+} \mathrm{e}^{\hat{S}} & =\hat{s}_{l}^{+} ; \\
\mathrm{e}^{-\hat{S}} \hat{s}_{l}^{z} \mathrm{e}^{\hat{S}} & =\hat{s}_{l}^{z}+a \hat{s}_{l}^{+} ; \\
\mathrm{e}^{-\hat{S}} \hat{s}_{l}^{-} \mathrm{e}^{\hat{S}} & =\hat{s}_{l}^{-}-2 a \hat{s}_{l}^{z}-a^{2} \hat{s}_{l}^{+},
\end{aligned}
$$

at the LSUB1 level of approximation. Thus we see that the corresponding CCM LSUB1 similarity transform of the Hamiltonian of Eq. (13) is given by

$$
\mathrm{e}^{-\hat{S}} \hat{H} \mathrm{e}^{\hat{S}}=-\sum_{i=1}^{N}\left(\hat{s}_{i}^{z} \hat{s}_{i+1}^{z}+a \hat{s}_{i}^{z} \hat{s}_{i+1}^{+}+a \hat{s}_{i}^{+} \hat{s}_{i+1}^{z}+a^{2} \hat{s}_{i}^{+} \hat{s}_{i+1}^{+}\right)
$$




$$
-\frac{\lambda}{2} \sum_{i=1}^{N}\left(\hat{s}_{i}^{+}+\hat{s}_{i}^{-}-2 a \hat{s}_{i}^{z}-a^{2} \hat{s}_{i}^{+}\right)
$$

By making use of the relation, $\hat{s}^{z}|\Phi\rangle=-\frac{1}{2}|\Phi\rangle$, for the present model state $|\Phi\rangle$, the groundstate energy is now readily evaluated from Eq. (9) as

$$
E_{g}=\left\langle\Phi\left|\mathrm{e}^{-\hat{S}} \hat{H} \mathrm{e}^{\hat{S}}\right| \Phi\right\rangle \Rightarrow \frac{E_{g}}{N}=-\frac{1}{4}-\frac{\lambda a}{2} .
$$

Furthermore the ground-state LSUB1 equation for the coefficient $a$ is given from Eq. (7) as follows,

$$
\frac{1}{N} \sum_{l=1}^{N}\left\langle\Phi\left|\hat{s}_{l}^{-} \mathrm{e}^{-\hat{S}} \hat{H} \mathrm{e}^{\hat{S}}\right| \Phi\right\rangle=0 \Rightarrow a-\frac{\lambda}{2}+\frac{\lambda a^{2}}{2}=0
$$

This quadratic equation has the physical solution

$$
a=\frac{1}{\lambda}\left(-1+\sqrt{\lambda^{2}+1}\right),
$$

where we have discarded the (unphysical) solution with the negative sign of the square root since $a$ must be zero when $\lambda=0$, as noted above. Using Eqs. (A4) and (A6), the ground-state energy is thus given by

$$
\frac{E_{g}}{N}=\frac{1}{4}-\frac{1}{2} \sqrt{\lambda^{2}+1}
$$

at the CCM LSUB1 level of approximation. As tests of this equation, we note that $E_{g} / N=$ $-1 / 4$ when $\lambda=0$ and that $E_{g} / N \rightarrow-\lambda / 2$ as $\lambda \rightarrow \infty$, which are the correct results in these two limiting cases. For comparison both with the exact result of Eq. (17) and with those from higher-order CCM LSUB $n$ approximations with $n>1$, the LSUB1 result of Eq. (A7) is also shown in Fig. 1.

At the same CCM LSUB1 level of approximation the bra-state $\hat{\tilde{S}}$ operator is given by

$$
\hat{\tilde{S}}=1+\tilde{a} \sum_{i=1}^{N} \hat{s}_{i}^{-}:
$$

such that we may now evaluate the LSUB1 ground-state energy expectation value functional, $\bar{H}$, as

$$
\bar{H} \equiv\langle\tilde{\Psi}|\hat{H}| \Psi\rangle=\left\langle\Phi\left|\hat{\tilde{S}} \mathrm{e}^{-\hat{S}} \hat{H} \mathrm{e}^{\hat{S}}\right| \Phi\right\rangle \Rightarrow \frac{1}{N} \bar{H}=-\frac{1}{4}-\frac{\lambda a}{2}+\tilde{a}\left(a-\frac{\lambda}{2}+\frac{\lambda a^{2}}{2}\right) .
$$

We see immediately that

$$
\frac{1}{N} \frac{\partial \bar{H}}{\partial \tilde{a}}=0 \Rightarrow a-\frac{\lambda}{2}+\frac{\lambda a^{2}}{2}=0,
$$

which simply gives us the LSUB1 ket-state equation of Eq. (A5) again, as required. Furthermore, we see that the LSUB1 bra-state coefficient $\tilde{a}$ can similarly now also be obtained as follows,

$$
\frac{1}{N} \frac{\partial \bar{H}}{\partial a}=0 \Rightarrow \tilde{a}-\frac{\lambda}{2}+\lambda a \tilde{a}=0 \Rightarrow \tilde{a}=\frac{\lambda}{2 \sqrt{\lambda^{2}+1}} .
$$


The magnetisation in the Ising direction,

$$
M^{z}=-\frac{1}{N} \sum_{i=1}^{N}\left\langle\tilde{\Psi}\left|\hat{s}_{i}^{z}\right| \Psi\right\rangle
$$

can now easily be evaluated at the LSUB1 level of approximation as

$$
M^{z}=\frac{1}{2}-\tilde{a} a=\frac{1}{2 \sqrt{\lambda^{2}+1}} .
$$

The transverse magnetisation is given by

$$
M^{\text {trans. }}=\frac{1}{N} \sum_{i=1}^{N}\left\langle\tilde{\Psi}\left|\hat{s}_{i}^{x}\right| \Psi\right\rangle=\frac{1}{2 N} \sum_{i=1}^{N}\left\langle\Psi\left|\left(\hat{s}_{i}^{+}+\hat{s}_{i}^{-}\right)\right| \Psi\right\rangle,
$$

which is readily evaluated at the LSUB1 level of approximation as

$$
M^{\text {trans. }}=\frac{a}{2}+\frac{\tilde{a}}{2}-\frac{\tilde{a} a^{2}}{2} .
$$

By substituting the explicit solutions for $a$ and $\tilde{a}$ from Eqs. (A6) and (A11), respectively, into Eq. (A15), we readily find the result

$$
M^{\text {trans. }}=\frac{\lambda}{2 \sqrt{\lambda^{2}+1}} .
$$

Once again, for comparison both with the corresponding exact results of Eqs. (18) and (19) and with those from higher-order CCM LSUB $n$ approximations with $n>1$, the LSUB1 results of Eqs. (A13) and (A16) are also shown in Figs. 2 and 3, respectively.

We now carry out a CCM LSUB1 calculation for the 1D transverse Ising model of Eq. (21), i.e., after the unitary transformation involving the rotation of the local spin axes. The Hamiltonian of Eq. (21) now contains terms with both real and imaginary coefficients, while the model state remains one in which all spins point in the downwards $z$-direction. We wish to compare LSUB1 results for this new Hamiltonian to those for the "unrotated" case with Hamiltonian given by Eq. (13). Indeed, we show explicitly that macroscopic quantities for this Hamiltonian do not change (and remain real), as they must, for the LSUB1 approximation even though the CCM correlation coefficients may now be complex-valued (i.e., contain real and imaginary components).

The LSUB1 approximation is again given by Eq. (A1) and the similarity transformed spin operators are given as before in Eq. (A2). Thus we see that

$$
\mathrm{e}^{-\hat{S}} \hat{H} \mathrm{e}^{\hat{S}}=-\sum_{i=1}^{N}\left(\hat{s}_{i}^{z} \hat{s}_{i+1}^{z}+a \hat{s}_{i}^{z} \hat{s}_{i+1}^{+}+a \hat{s}_{i}^{+} \hat{s}_{i+1}^{z}+a^{2} \hat{s}_{i}^{+} \hat{s}_{i+1}^{+}\right)-\frac{\mathrm{i} \lambda}{2} \sum_{i=1}^{N}\left(-\hat{s}_{i}^{+}+\hat{s}_{i}^{-}-2 a \hat{s}_{i}^{z}-a^{2} \hat{s}_{i}^{+}\right) .
$$

The ground-state energy equation is now given by

$$
E_{g}=\left\langle\Phi\left|\mathrm{e}^{-\hat{S}} \hat{H} \mathrm{e}^{\hat{S}}\right| \Phi\right\rangle \Rightarrow \frac{E_{g}}{N}=-\frac{1}{4}-\frac{\lambda a \mathrm{i}}{2},
$$

and the ground-state energy LSUB1 equation is given by

$$
\frac{1}{N} \sum_{l=1}^{N}\left\langle\Phi\left|\hat{s}_{l}^{-} \mathrm{e}^{-\hat{S}} \hat{H} \mathrm{e}^{\hat{S}}\right| \Phi\right\rangle=0 \Rightarrow a+\frac{\mathrm{i} \lambda}{2}+\frac{\mathrm{i} \lambda a^{2}}{2}=0 .
$$


Again, this quadratic equation has the physical solution given by

$$
a=\frac{\mathrm{i}}{\lambda}\left(1-\sqrt{\lambda^{2}+1}\right),
$$

where we have now discarded the (unphysical) solution with the positive sign of the square root since $a$ must be zero when $\lambda=0$, as noted previously. Thus, we see that the new LSUB1 ket-state correlation coefficient $a$ is now complex-valued (indeed, pure imaginary) for all values of $\lambda(>0)$. Substitution of this value for $a$ from Eq. (A20) into Eq. (A18) immediately yields that the ground-state energy at the CCM LSUB1 level of approximation is given by

$$
\frac{E_{g}}{N}=\frac{1}{4}-\frac{1}{2} \sqrt{\lambda^{2}+1}
$$

which is indeed seen to be identical to that given by Eq. (A7). The bra-state $S$ operator is again given by Eq. (A8), such that we may explicitly evaluate the ground-state energy functional,

$$
\bar{H} \equiv\langle\tilde{\Psi}|\hat{H}| \Psi\rangle=\left\langle\Phi\left|\hat{\tilde{S}} \mathrm{e}^{-\hat{S}} \hat{H} \mathrm{e}^{\hat{S}}\right| \Phi\right\rangle \Rightarrow \frac{1}{N} \bar{H}=-\frac{1}{4}-\frac{\mathrm{i} \lambda a}{2}+\tilde{a}\left(a+\frac{\mathrm{i} \lambda}{2}+\frac{\mathrm{i} \lambda a^{2}}{2}\right) .
$$

We see immediately that

$$
\frac{1}{N} \frac{\partial \bar{H}}{\partial \tilde{a}}=0 \Rightarrow a+\frac{\mathrm{i} \lambda}{2}+\frac{\mathrm{i} \lambda a^{2}}{2}=0
$$

again as required. Furthermore, we see that

$$
\frac{1}{N} \frac{\partial \bar{H}}{\partial a}=0 \Rightarrow \tilde{a}-\frac{\mathrm{i} \lambda}{2}+\mathrm{i} \lambda a \tilde{a}=0 \Rightarrow \tilde{a}=\frac{\mathrm{i} \lambda}{2 \sqrt{\lambda^{2}+1}} .
$$

The magnetisation in the Ising direction is again given by Eq. (A12), which at the LSUB1 level of approximation (now using Eqs. (A20) and (A24)) is given by

$$
M^{z}=\frac{1}{2}-a \tilde{a}=\frac{1}{2 \sqrt{\lambda^{2}+1}},
$$

which is now explicitly real and in agreement with Eq. (A13), both as required. The transverse magnetisation should now, of course, be evaluated with respect to the $y$-direction (in terms of spin coordinates after rotation of the local spin axes) and it is given by

$$
M^{\text {trans. }}=\frac{1}{N} \sum_{i=1}^{N}\left\langle\tilde{\Psi}\left|\hat{s}_{i}^{y}\right| \Psi\right\rangle=\frac{\mathrm{i}}{2 N} \sum_{i=1}^{N}\left\langle\Psi\left|\left(\hat{s}_{i}^{-}-\hat{s}_{i}^{+}\right)\right| \Psi\right\rangle,
$$

which at the LSUB1 level of approximation, as defined by Eqs. (A1) and (A8), is readily evaluated as

$$
M^{\text {trans. }}=\frac{\mathrm{i} a}{2}-\frac{\mathrm{i} \tilde{a}}{2}-\frac{\mathrm{i} \tilde{a} a^{2}}{2} .
$$

Direct substitution into Eq. (A27) with the LSUB1 solutions for $a$ and $\tilde{a}$ from Eqs. (A20) and (A24), respectively, readily yields the explicit expression,

$$
M^{\text {trans. }}=\frac{\lambda}{2 \sqrt{\lambda^{2}+1}} .
$$

which is again explicitly real and in agreement with Eq. (A16), both as required.

Thus, we have explicitly shown that the results for the ground-state energy per spin and the magnetisations in the Ising and transverse directions at the LSUB1 level of approximation for 
the Hamiltonian of Eq. (21), which contains complex-valued coefficients, are not only real numbers, even though both the ket- and bra-states correlation coefficients are demonstrably complex-valued, but they are also identical to the corresponding results derived previously for the unitarily equivalent Hamiltonian of Eq. (13).

\section{Appendix B: Calculation of the Rotated Hamiltonian for Model State V}

We start from a Hamiltonian given by Eq. (23), i.e.,

$$
\hat{H}=\sum_{\langle i, j\rangle} \hat{\mathbf{s}}_{i} \cdot \hat{\mathbf{s}}_{j}-\lambda \sum_{i=1}^{N} \hat{s}_{i}^{z}=\sum_{\langle i, j\rangle}\left(\hat{s}_{i}^{x} \hat{s}_{j}^{x}+\hat{s}_{i}^{y} \hat{s}_{j}^{y}+\hat{s}_{i}^{z} \hat{s}_{j}^{z}\right)-\lambda \sum_{i=1}^{N} \hat{s}_{i}^{z},
$$

where the sum over the index $\langle i, j\rangle$ indicate a sum over all nearest-neighbour pairs on the triangular lattice, with each pair being counted once and once only. We now carry out the first of a number of unitary transformations of the local spin axes given by,

$$
\hat{s}_{i}^{x} \rightarrow \hat{s}_{i}^{x} ; \quad \hat{s}_{i}^{y} \rightarrow-\hat{s}_{i}^{z} ; \quad \hat{s}_{i}^{z} \rightarrow \hat{s}_{i}^{y},
$$

where $i$ runs over all lattice sites on the triangular lattice. The Hamiltonian is now given by

$$
\hat{H}=\sum_{\langle i, j\rangle}\left(\hat{s}_{i}^{x} \hat{s}_{j}^{x}+\hat{s}_{i}^{y} \hat{s}_{j}^{y}+\hat{s}_{i}^{z} \hat{s}_{j}^{z}\right)-\lambda \sum_{i=1}^{N} \hat{s}_{i}^{y},
$$

This first transformation represents a rotation by $90^{\circ}$ about the $x$-axis. We do this so that the subsequent rotations of the model state are both slightly easier to formulate and follow in direct analogy to earlier work on the coplanar states for this model. In particular, the next set of rotations of spins in the $x z$-plane follow exactly that set out in [21] for the triangular lattice with zero external field. We first define three interpenetrating sublattices $(A, B, C)$ for the triangular lattice, such that each elementary triangular plaquette formed from nearestneighbour sites of the original lattice contains one site from each of the three sublattices. Hence, we start now with the the usual $120^{\circ}$ three-sublattice Néel state, as shown for state I in Fig. 4, with $\alpha=30^{\circ}$. The spins all lie in the $x z$-plane, with spins on sublattice $C$ aligned along the negative $z$-axis and those on sublattices $A$ and $B$ oriented respectively at angles $-120^{\circ}$ and $+120^{\circ}$ with respect to those on sublattice $C$. We now perform the necessary passive rotations so that all spins point downwards (i.e., in the negative $z$-direction). Thus, we rotate the axes for spins at sites $i_{A}$ on the $A$-sublattice by $+120^{\circ}$ about the $y$-axis via:

$$
\hat{s}_{i_{A}}^{x} \rightarrow-\frac{1}{2} \hat{s}_{i_{A}}^{x}-\frac{\sqrt{3}}{2} \hat{s}_{i_{A}}^{z} ; \quad \hat{s}_{i_{A}}^{y} \rightarrow \hat{s}_{i_{A}}^{y} ; \quad \hat{s}_{i_{A}}^{z} \rightarrow \frac{\sqrt{3}}{2} \hat{s}_{i_{A}}^{x}-\frac{1}{2} \hat{s}_{i_{A}}^{z} .
$$

Simultaneously, we rotate the axes for spins at sites $i_{B}$ on the $B$-sublattice by $-120^{\circ}$ about the $y$-axis via:

$$
\hat{s}_{i_{B}}^{x} \rightarrow-\frac{1}{2} \hat{s}_{i_{B}}^{x}+\frac{\sqrt{3}}{2} \hat{s}_{i_{B}}^{z} ; \quad \hat{s}_{i_{B}}^{y} \rightarrow \hat{s}_{i_{B}}^{y} ; \quad \hat{s}_{i_{B}}^{z} \rightarrow-\frac{\sqrt{3}}{2} \hat{s}_{i_{B}}^{x}-\frac{1}{2} \hat{s}_{i_{B}}^{z} .
$$

Furthermore, in this step, we do not rotate the axes for spins at sites $i_{C}$ on the $C$-sublattice, since they are already pointing in the downwards direction:

$$
\hat{s}_{i_{C}}^{x} \rightarrow \hat{s}_{i_{C}}^{x} ; \quad \hat{s}_{i_{C}}^{y} \rightarrow \hat{s}_{i_{C}}^{y} ; \quad \hat{s}_{i_{C}}^{z} \rightarrow \hat{s}_{i_{C}}^{z} .
$$


We may now use Eqs. (B4)-(B6) to rewrite the nearest-neighbour part of the Hamiltonian of Eq. (B3) that connects sites $i_{B}$ on the $B$-sublattice and $j_{C}$ on the $C$-sublattice in the direction going from site $i_{B}$ to site $j_{C}$ in the (unitarily equivalent) rotated form

$$
\begin{aligned}
\hat{H}_{i_{B} \rightarrow j_{C}} & \equiv \sum_{\left\langle i_{B} \rightarrow j_{C}\right\rangle}\left\{\hat{s}_{i_{B}}^{x} \hat{s}_{j_{C}}^{x}+\hat{s}_{i_{B}}^{y} \hat{s}_{j_{C}}^{y}+\hat{s}_{i_{B}}^{z} \hat{s}_{j_{C}}^{z}\right\} \\
& =\sum_{\left\langle i_{B} \rightarrow j_{C}\right\rangle}\left\{\left(-\frac{1}{2} \hat{s}_{i_{B}}^{x}+\frac{\sqrt{3}}{2} \hat{s}_{i_{B}}^{z}\right) \hat{s}_{j_{C}}^{x}+\hat{s}_{i_{B}}^{y} \hat{s}_{j_{C}}^{y}+\left(-\frac{\sqrt{3}}{2} \hat{s}_{i_{B}}^{x}-\frac{1}{2} \hat{s}_{i_{B}}^{z}\right) \hat{s}_{j_{C}}^{z}\right\} \\
& =\sum_{\left\langle i_{B} \rightarrow j_{C}\right\rangle}\left\{-\frac{1}{2} \hat{s}_{i_{B}}^{x} \hat{s}_{j_{C}}^{x}+\frac{\sqrt{3}}{2}\left(\hat{s}_{i_{B}}^{z} \hat{s}_{j_{C}}^{x}-\hat{s}_{i_{B}}^{x} \hat{s}_{j_{C}}^{z}\right)+\hat{s}_{i_{B}}^{y} \hat{s}_{j_{C}}^{y}-\frac{1}{2} \hat{s}_{i_{B}}^{z} \hat{s}_{j_{C}}^{z}\right\} .
\end{aligned}
$$

We may similarly use Eqs. (B4)-(B6) to rewrite the nearest-neighbour part of the Hamiltonian of Eq. (B3) that connects sites $i_{C}$ on the $C$-sublattice and $j_{A}$ on the $A$-sublattice in the direction going from site $i_{C}$ to site $j_{A}$ in the rotated form

$$
\begin{aligned}
\hat{H}_{i_{C} \rightarrow j_{A}} & \equiv \sum_{\left\langle i_{C} \rightarrow j_{A}\right\rangle}\left\{\hat{s}_{i_{C}}^{x} \hat{s}_{j_{A}}^{x}+\hat{s}_{i_{C}}^{y} \hat{s}_{j_{A}}^{y}+\hat{s}_{i_{C}}^{z} \hat{s}_{j_{A}}^{z}\right\} \\
& =\sum_{\left\langle i_{C} \rightarrow j_{A}\right\rangle}\left\{\hat{s}_{i_{C}}^{x}\left(-\frac{1}{2} \hat{s}_{j_{A}}^{x}-\frac{\sqrt{3}}{2} \hat{s}_{j_{A}}^{z}\right)+\hat{s}_{i_{C}}^{y} \hat{s}_{j_{A}}^{y}+\hat{s}_{j_{A}}^{z}\left(\frac{\sqrt{3}}{2} \hat{s}_{j_{A}}^{x}-\frac{1}{2} \hat{s}_{j_{A}}^{z}\right)\right\} \\
& =\sum_{\left\langle i_{C} \rightarrow j_{A}\right\rangle}\left\{-\frac{1}{2} \hat{s}_{i_{C}}^{x} \hat{s}_{j_{A}}^{x}+\frac{\sqrt{3}}{2}\left(\hat{s}_{i_{C}}^{z} \hat{s}_{j_{A}}^{x}-\hat{s}_{i_{C}}^{x} \hat{s}_{j_{A}}^{z}\right)+\hat{s}_{i_{C}}^{y} \hat{s}_{j_{A}}^{y}-\frac{1}{2} \hat{s}_{i_{C}}^{z} \hat{s}_{j_{A}}^{z}\right\} .
\end{aligned}
$$

Lastly, we again use Eqs. (B4)-(B6) to rewrite the nearest-neighbour part of the Hamiltonian of Eq. (B3) that connects sites $i_{A}$ on the $A$-sublattice and $j_{B}$ on the $B$-sublattice in the direction going from site $i_{A}$ to site $j_{B}$ in the rotated form

$$
\begin{aligned}
\hat{H}_{i_{A} \rightarrow j_{B}} \equiv & \sum_{\left\langle i_{A} \rightarrow j_{B}\right\rangle}\left\{\hat{s}_{i_{A}}^{x} \hat{s}_{j_{B}}^{x}+\hat{s}_{i_{A}}^{y} \hat{s}_{j_{B}}^{y}+\hat{s}_{i_{A}}^{z} \hat{s}_{j_{B}}^{z}\right\} \\
= & \sum_{\left\langle i_{A} \rightarrow j_{B}\right\rangle}\left\{\left(-\frac{1}{2} \hat{s}_{i_{A}}^{x}-\frac{\sqrt{3}}{2} \hat{s}_{i_{A}}^{z}\right)\left(-\frac{1}{2} \hat{s}_{j_{B}}^{x}+\frac{\sqrt{3}}{2} \hat{s}_{j_{B}}^{z}\right)+\hat{s}_{i_{A}}^{y} \hat{s}_{j_{B}}^{y}\right. \\
& \left.+\left(\frac{\sqrt{3}}{2} \hat{s}_{i_{A}}^{x}-\frac{1}{2} \hat{s}_{i_{A}}^{z}\right)\left(-\frac{\sqrt{3}}{2} \hat{s}_{j_{B}}^{x}-\frac{1}{2} \hat{s}_{j_{B}}^{z}\right)\right\} \\
= & \sum_{\left\langle i_{A} \rightarrow j_{B}\right\rangle}\left\{-\frac{1}{2} \hat{s}_{i_{A}}^{x} \hat{s}_{j_{B}}^{x}+\frac{\sqrt{3}}{2}\left(\hat{s}_{i_{A}}^{z} \hat{s}_{j_{B}}^{x}-\hat{s}_{i_{A}}^{x} \hat{s}_{j_{B}}^{z}\right)+\hat{s}_{i_{A}}^{y} \hat{s}_{j_{B}}^{y}-\frac{1}{2} \hat{s}_{i_{A}}^{z} \hat{s}_{j_{B}}^{z}\right\} .
\end{aligned}
$$

By making use of Eqs. (B7)-(B9), the Hamiltonian of Eq. (B3) may now be written as

$$
\begin{aligned}
\hat{H}= & \sum_{\left\langle i_{B, C} A \rightarrow j_{C, A, B}\right\rangle}\left\{-\frac{1}{2} \hat{s}_{i_{B, C, A}}^{x} \hat{s}_{j_{C, A, B}}^{x}+\frac{\sqrt{3}}{2}\left(\hat{s}_{i_{B, C, A}}^{z} \hat{s}_{j_{C, A, B}}^{x}-\hat{s}_{i_{B, C, A}}^{x} \hat{s}_{j_{C, A, B}}^{z}\right)\right. \\
& \left.+\hat{s}_{i_{B, C, A}}^{y} \hat{s}_{j_{C, A, B}}^{y}-\frac{1}{2} \hat{s}_{i_{B, C, A}}^{z} \hat{s}_{j_{C, A, B}}^{z}\right\}-\lambda \sum_{i=1}^{N} \hat{s}_{i}^{y},
\end{aligned}
$$

after the second set of rotations of the local spin axes have been made, and where the sum over $\left\langle i_{B, C, A} \rightarrow j_{C, A, B}\right\rangle$ represent a shorthand notation to include the three sorts of "directed" nearest-neighbour bonds on each basic triangular plaquette of side $a$ on the triangular lattice, 
which join sites $i_{B}$ and $j_{C}$ going from the $B$-sublattice to the $C$-sublattice, sites $i_{C}$ and $j_{A}$ going from the $C$-sublattice to the $A$-sublattice, and sites $i_{A}$ and $j_{B}$ going from the $A$-sublattice to the $B$-sublattice (in those directions only and not reversed).

The effects of an external field for this model state can then be included straightforwardly by a final rotation in the $y z$-plane (i.e., about the $x$-axis) by an angle of $\theta$ for all spins (i.e., on all sublattices). This is performed via the following transformation:

$$
\hat{s}_{i}^{x} \rightarrow \hat{s}_{i}^{x} ; \quad \hat{s}_{i}^{y} \rightarrow \cos \theta \hat{s}_{i}^{y}-\sin \theta \hat{s}_{i}^{z} ; \quad \hat{s}_{i}^{z} \rightarrow \sin \theta \hat{s}_{i}^{y}+\cos \theta \hat{s}_{i}^{z} .
$$

The Hamiltonian of Eq. (B10) is thus now

$$
\begin{aligned}
& \hat{H}= \sum_{\left\langle i_{B, C, A} \rightarrow j_{C, A, B}\right\rangle}\left\{\frac { \sqrt { 3 } } { 2 } \left[\left(\sin \theta \hat{s}_{i_{B, C, A}}^{y}+\cos \theta \hat{s}_{i_{B, C, A}}^{z}\right) \hat{s}_{j_{C, A, B}}^{x}\right.\right. \\
&\left.-\hat{s}_{i_{B, C, A}}^{x}\left(\sin \theta \hat{s}_{j_{C, A, B}}^{y}+\cos \theta \hat{s}_{j_{C, A, B}}^{z}\right)\right] \\
&-\frac{1}{2} \hat{s}_{i_{B, C, A}}^{x} \hat{s}_{j_{C, A, B}}^{x}+\left(\cos \theta \hat{s}_{i_{B, C, A}}^{y}-\sin \theta \hat{s}_{i_{B, C, A}}^{z}\right)\left(\cos \theta \hat{s}_{j_{C, A, B}}^{y}-\sin \theta \hat{s}_{j_{C, A, B}}^{z}\right) \\
&\left.-\frac{1}{2}\left(\sin \theta \hat{s}_{i_{B, C, A}}^{y}+\cos \theta \hat{s}_{i_{B, C, A}}^{z}\right)\left(\sin \theta \hat{s}_{j_{C, A, B}}^{y}+\cos \theta \hat{s}_{j_{C, A, B}}^{z}\right)\right\} \\
&-\lambda \sum_{i=1}^{N}\left[\cos \theta \hat{s}_{i}^{y}-\sin \theta \hat{s}_{i}^{z}\right] \\
&\left\langle\sum _ { i _ { B , C , A } \rightarrow j _ { C , A , B } \rangle } \left\{\left(-\frac{1}{2} \cos { }^{2} \theta+\sin ^{2} \theta\right) \hat{s}_{i_{B, C, A}}^{z} \hat{s}_{j_{C, A, B}}^{z}-\frac{1}{2} \hat{s}_{i_{B, C, A}}^{x} \hat{s}_{j_{C, A, B}}^{x}\right.\right. \\
&+\left(-\frac{1}{2} \sin { }^{2} \theta+\cos ^{2} \theta\right) \hat{s}_{i_{B, C, A}}^{y} \hat{s}_{j_{C, A, B}}^{y} \\
&+\frac{\sqrt{3}}{2} \cos \theta\left(\hat{s}_{i_{B, C, A}}^{z} \hat{s}_{j_{C, A, B}}^{x}-\hat{s}_{i_{B, C, A}}^{x} \hat{s}_{j_{C, A, B}}^{z}\right) \\
&+\frac{\sqrt{3}}{2} \sin \theta\left(\hat{s}_{i_{B, C, A}}^{y} \hat{s}_{j_{C, A, B}}^{x}-\hat{s}_{i_{B, C, A}}^{x} \hat{s}_{j_{C, A, B}}^{y}\right) \\
&\left.-\frac{3}{2} \sin \theta \cos \theta\left(\hat{s}_{i_{B, C, A}}^{y} \hat{s}_{j_{C, A, B}}^{z}+\hat{s}_{i_{B, C, A}}^{z} \hat{s}_{j_{C, A, B}}^{y}\right)\right\} \\
&-\lambda \sum_{i=1}^{N}\left[\cos \theta \hat{s}_{i}^{y}-\sin \theta \hat{s}_{i}^{z}\right] . \\
&
\end{aligned}
$$

As shown in Fig. 5, we obtain a minimal energy solution for $\theta=0$ (i.e., where all spins lie in the $x z$-plane) when the external field $\lambda$ is zero, whereas we obtain a minimal energy solution for $\theta=\pi / 2$ (i.e., where all spins point along the $y$-axis) when $\lambda$ reaches the saturation field, $\lambda_{s}=4.5$, for the spin- $\frac{1}{2}$ model. Equation (B12) may then be rewritten in our final form,

$$
\begin{aligned}
\hat{H}= & \sum_{\left\langle i_{B, C, A} \rightarrow j_{C, A, B}\right\rangle}\left\{\left(\sin ^{2} \theta-\frac{1}{2} \cos ^{2} \theta\right) \hat{s}_{i_{B, C, A}}^{z} \hat{s}_{j_{C, A, B}}^{z}\right. \\
& +\frac{1}{4}\left(\frac{1}{2} \sin ^{2} \theta-\cos ^{2} \theta-\frac{1}{2}\right)\left(\hat{s}_{i_{B, C, A}}^{+} \hat{s}_{j_{C, A, B}}^{+}+\hat{s}_{i_{B, C, A}^{-}}^{-} \hat{s}_{j_{C, A, B}}^{-}\right) \\
& +\frac{1}{4}\left(\cos ^{2} \theta-\frac{1}{2} \sin ^{2} \theta-\frac{1}{2}\right)\left(\hat{s}_{i_{B, C, A}}^{+} \hat{s}_{j_{C, A, B}}^{-}+\hat{s}_{i_{B, C, A}}^{-} \hat{s}_{j_{C, A, B}}^{+}\right)
\end{aligned}
$$




$$
\begin{aligned}
& \left.+\frac{\sqrt{3}}{4} \cos \theta\left[\hat{s}_{i_{B, C, A}}^{z}\left(\hat{s}_{j_{C, A, B}}^{+}+\hat{s}_{j_{C, A, B}}^{-}\right)-\left(\hat{s}_{i_{B, C, A}}^{+}+\hat{s}_{i_{B, C, A}}^{-}\right) \hat{s}_{j_{C, A, B}}^{z}\right]\right\} \\
& +\lambda \sum_{i=1}^{N} \sin \theta \hat{s}_{i}^{z} \\
& +\mathrm{i} \sum_{\left\langle i_{B, C, A} \rightarrow j_{C, A, B}\right\rangle}\left\{\frac{\sqrt{3}}{4} \sin \theta\left(\hat{s}_{i_{B, C, A}}^{-} \hat{s}_{j_{C, A, B}}^{+}-\hat{s}_{i_{B, C, A}}^{+} \hat{s}_{j_{C, A, B}}^{-}\right)\right. \\
& \left.+\frac{3}{4} \sin \theta \cos \theta\left[\hat{s}_{i_{B, C, A}}^{z}\left(\hat{s}_{j_{C, A, B}}^{+}-\hat{s}_{j_{C, A, B}^{-}}^{-}\right)+\left(\hat{s}_{i_{B, C, A}}^{+}-\hat{s}_{i_{B, C, A}}^{-}\right) \hat{s}_{j_{C, A, B}}^{z}\right]\right\} \\
& +\mathrm{i} \frac{\lambda}{2} \sum_{i=1}^{N} \cos \theta\left(\hat{s}_{i}^{+}-\hat{s}_{i}^{-}\right) .
\end{aligned}
$$

\section{References}

1. Coester, F.: Bound states of a many-particle system. Nucl. Phys. 7, 421 (1958)

2. Coester, F., Kümmel, H.: Short-range correlations in nuclear wave functions. Nucl. Phys. 17, 477 (1960)

3. Čižek, J.: On the correlation problem in atomic and molecular systems. Calculation of wavefunction components in Ursell-type expansion using quantum-field theoretical methods. J. Chem. Phys. 45, 4256 (1966)

4. Čǐ̌ek, J.: On the use of the cluster expansion and the technique of diagrams in calculations of correlation effects in atoms and molecules. Adv. Chem. Phys. 14, 35 (1969)

5. Bishop, R.F., Lührmann, K.H.: Electron correlations: I. Ground-state results in the high-density regime. Phys. Rev. B 17, 3757 (1978)

6. Bishop, R.F., Lührmann, K.H.: Electron correlations. II. Ground-state results at low and metallic densities. Phys. Rev. B 26, 5523 (1982)

7. Kümmel, H., Lührmann, K.H., Zabolitzky, J.G.: Many-fermion theory in expS-(or coupled cluster) form. Phys. Rep. 36C, 1 (1978)

8. Arponen, J.S.: Variational principles and linked-cluster exp S expansions for static and dynamic manybody problems. Ann. Phys. (N.Y.) 151, 311 (1983)

9. Bishop, R.F., Kümmel, H.: The coupled-cluster method. Phys. Today 40(3), 52 (1987)

10. Arponen, J.S., Bishop, R.F., Pajanne, E.: Extended coupled-cluster method. I. Generalized coherent bosonization as a mapping of quantum theory into classical Hamiltonian mechanics. Phys. Rev. A 36, 2519 (1987)

11. Arponen, J.S., Bishop, R.F., Pajanne, E.: Extended coupled-cluster method. II. Excited states and generalized random-phase approximation. Phys. Rev. A 36, 2539 (1987)

12. Arponen, J.S., Bishop, R.F., Pajanne, E.: In: Vashishta, P., Kalia, R.K., Bishop, R.F. (eds.) Condensed Matter Theories, vol. 2, p. 357. Plenum, New York (1987)

13. Bartlett, R.J.: Coupled-cluster approach to molecular structure and spectra: a step toward predictive quantum chemistry. J. Phys. Chem. 93, 1697 (1989)

14. Bishop, R.F.: An overview of coupled cluster theory and its applications in physics. Theor. Chim. Acta 80, 95 (1991)

15. Bishop, R.F.: The coupled cluster method. In: Navarro, J., Polls, A. (eds.) Microscopic Quantum ManyBody Theories and Their Applications. Lecture Notes in Physics, vol. 510, p. 1. Springer, Berlin (1998)

16. Roger, M., Hetherington, J.H.: Coupled-cluster approximation for spin lattices: application to solid $\mathrm{He}_{3}$. Phys. Rev. B 41, 200 (1990)

17. Roger, M., Hetherington, J.H.: The coupled-cluster approximation: a simple approach to spin-lattice and Hubbard models. Europhys. Lett. 11, 255 (1990)

18. Bishop, R.F., Parkinson, J.B., Xian, Y.: Coupled-cluster treatments of correlations in quantum antiferromagnets. Phys. Rev. B 44, 9425 (1991)

19. Bishop, R.F., Parkinson, J.B., Xian, Y.: Coupled-cluster calculations of quantum XXZ models with a general spin. Phys. Rev. B 46, 880 (1992)

20. Xian, Y.: A microscopic approach to the dimerization in frustrated spin-1/2 antiferromagnetic chains. J. Phys. Condens. Matter 6, 5965 (1994) 
21. Zeng, C., Farnell, D.J.J., Bishop, R.F.: An efficient implementation of high-order coupled-cluster techniques applied to quantum magnets. J. Stat. Phys. 90, 327 (1998)

22. Bishop, R.F., Farnell, D.J.J., Krüger, S.E., Parkinson, J.B., Richter, J., Zeng, C.: High-order coupled cluster method calculations for the ground-and excited-state properties of the spin-half XXZ model. J. Phys. Condens. Matter 12, 6887 (2000)

23. Farnell, D.J.J., Bishop, R.F., Gernoth, K.A.: Coupled cluster method calculations of quantum magnets with spins of general spin quantum number. J. Stat. Phys. 108, 401 (2002)

24. Farnell, D.J.J., Bishop, R.F.: The coupled cluster method applied to quantum magnetism. In: Schollwöck, U., Richter, J., Farnell, D.J.J., Bishop, R.F. (eds.) Quantum Magnetism, p. 307. Springer, Berlin (2004)

25. Farnell, D.J.J., Schulenberg, J., Richter, J., Gernoth, K.A.: High-order coupled cluster calculations via parallel processing: an illustration for $\mathrm{CaV}_{4} \mathrm{O}_{9}$. Phys. Rev. B 72, 172408 (2005)

26. Darradi, R., Richter, J., Farnell, D.J.J.: Coupled cluster treatment of the Shastry-Sutherland antiferromagnet. Phys. Rev. B 72, 104425 (2005)

27. Krüger, S., Darradi, R., Richter, J., Farnell, D.J.J.: Direct calculation of the spin stiffness of the spin-1/2 Heisenberg antiferromagnet on square, triangular, and cubic lattices using the coupled-cluster method. Phys. Rev. B 73, 094404 (2006)

28. Darradi, R., Derzhko, O., Zinke, R., Schulenburg, J., Krüger, S.E., Richter, J.: Ground state phases of the spin-1/2 $\mathrm{J}_{1}-\mathrm{J}_{2}$ Heisenberg antiferromagnet on the square lattice: a high-order coupled cluster treatment. Phys. Rev. B 78, 214415 (2008)

29. Farnell, D.J.J., Richter, J., Zinke, R., Bishop, R.F.: High-order coupled cluster method (CCM) calculations for quantum magnets with valence-bond ground states. J. Stat. Phys. 135, 175 (2009)

30. Farnell, D.J.J., Richter, J., Zinke, R.: High-order coupled cluster method study of frustrated and unfrustrated quantum magnets in external magnetic fields. J. Phys. Condens. Matter 21, 406002 (2009)

31. Farnell, D.J.J., Croudace, A.I.: High-Order Coupled Cluster Method Calculations using ThreeDimensional Model States: An Illustration for the Triangular-Lattice Antiferromagnet in an External Field. eprint arXiv: 1312.0785

32. Farnell, D.J.J., Bishop, R.F., Li, P.H.Y., Richter, J., Campbell, C.E.: The frustrated Heisenberg antiferromagnet on the honeycomb lattice: a candidate for deconfined quantum criticality. Phys. Rev. B 84, 012403 (2011)

33. Götze, O., Farnell, D.J.J., Bishop, R.F., Li, P.H.Y., Richter, J.: Heisenberg antiferromagnet on the kagome lattice with arbitrary spin: a high-order coupled cluster treatment. Phys. Rev. B 84, 224428 (2011)

34. Farnell, D.J.J., Darradi, R., Schmidt, R., Richter, J.: Spin-half Heisenberg antiferromagnet on two archimedian lattices: from the bounce lattice to the maple-leaf lattice and beyond. Phys. Rev. B 84, 104406 (2011)

35. Li, P.H.Y., Bishop, R.F., Farnell, D.J.J., Richter, J., Campbell, C.E.: Ground-state phases of the frustrated spin-1/2 $J_{1}-J_{2}-J_{3}$ Heisenberg ferromagnet $(\mathrm{J} 1<0)$ on the honeycomb lattice with $J_{3}=J_{2}>0$. Phys. Rev. B 85, 085115 (2012)

36. Richter, J., Götze, O., Zinke, R., Farnell, D.J.J., Tanaka, H.: The magnetization process of the spin-one triangular-lattice Heisenberg antiferromagnet. J. Phys. Soc. Jpn. 82, 015002 (2013)

37. Farnell, D.J.J., Götze, O., Richter, J., Bishop, R.F., Li, P.H.Y.: Quantum $s=1 / 2$ antiferromagnets on archimedean lattices: The route from semiclassical magnetic order to nonmagnetic quantum states. Phys. Rev. B 89, 184407 (2014)

38. Götze, O., Richter, J.: Ground-state phase diagram of the XXZ spin-s kagome antiferromagnet: a coupledcluster study. Phys. Rev. B 91, 104402 (2015)

39. Götze, O., Richter, J.: The route to magnetic order in the spin-1/2 kagome Heisenberg antiferromagnet: the role of interlayer coupling. Europhys. Lett. (EPL) 114, 67004 (2016)

40. Farnell, D.J.J., Götze, O., Richter, J.: Ground-state ordering of the $J_{1}-J_{2}$ model on the simple cubic and body-centered cubic lattices. Phys. Rev. B 93, 235123 (2016)

41. Bishop, R.F., Li, P.H.Y.: High-order study of the quantum critical behavior of a frustrated spin-1/2 antiferromagnet on a stacked honeycomb bilayer. Phys. Rev. B 96, 224416 (2017)

42. Bishop, R.F., Li, P.H.Y.: Low-energy parameters and spin gap of a frustrated spin-s Heisenberg antiferromagnet with $s \leq \frac{3}{2}$ on the honeycomb lattice. J. Phys. Conf. Ser. 1041, 012001 (2018)

43. We consider here the "standard" program package CCCM of D.J.J. Farnell and J. Schulenburg, see http:// www-e.uni-magdeburg.de/jschulen/ccm/index.html

44. Pfeuty, P.: The one-dimensional Ising model with a transverse field. Ann. Phys. (N.Y.) 57, 79 (1970)

45. Kawamura, H., Miyashita, S.: Phase transition of the Heisenberg antiferromagnet on the triangular lattice in a magnetic field. J. Phys. Soc. Jpn. 54, 4530 (1985)

46. Chubukov, A.V., Golosov, D.I.: Quantum theory of an antiferromagnet on a triangular lattice in a magnetic field. J. Phys. Condens. Matter 3, 69 (1991) 
47. Gvozdikova, M.V., Melchy, P.E., Zhitomirsky, M.E.: Magnetic phase diagrams of classical triangular and kagome antiferromagnets. J. Phys. Condens. Matter 23, 164209 (2001)

48. Petrenko, O.A., Honecker, A., Zhitomirsky, M.E.: Field induced ordering in the frustrated square-lattice antiferromagnet. Phys. Rev. Lett. 85, 3269 (2000)

49. Zhitomirsky, M.E.: Field-induced transitions in a Kagomé antiferromagnet. Phys. Rev. Lett. 88, 057204 (2002)

50. Nishimori, H., Miyashita, S.: Magnetization process of the spin-1/2 antiferromagnetic Ising-like Heisenberg model on the triangular lattice. J. Phys. Soc. Jpn. 55, 4448 (1986)

51. Bernu, B., Lecheminant, P., Lhuillier, C., Pierre, L.: Exact spectra, spin susceptibilities, and order parameter of the quantum Heisenberg antiferromagnet on the triangular lattice. Phys. Rev. B 50, 10048 (1994)

52. Honecker, A.: A comparative study of the magnetization process of two-dimensional antiferromagnets. J. Phys. Condens. Matter 11, 4697 (1999)

53. Cabra, D.C., Grynberg, M.D., Honecker, A., Pujol, P.: Magnetization plateaux in quasi-one-dimensional strongly correlated electron systems: a brief review. In: Hernández, S., Clark, J.W. (eds.) Condensed Matter Theories, vol. 16, p. 17. Nova Science Publications, New York (2001). eprint arXiv:cond-mat/0010376

54. Lhuillier, C., Misguich, G.: Frustrated quantum magnetism. In: Berthier, C., Lévy, L.P., Martinez, G. (eds.) High Magnetic Fields. Lecture Notes in Physics, vol. 595, p. 161. Springer, Berlin (2002)

55. Yoshikawa, S., Okunishi, K., Senda, M., Miyashita, S.: Quantum fluctuation-induced phase transition in $S=1 / 2$ XY-like Heisenberg antiferromagnets on the triangular lattice. J. Phys. Soc. Jpn. 73, 1798 (2004)

56. Richter, J., Schulenburg, J., Honecker, A.: Quantum magnetism in two dimensions: from semi-classical Néel order to magnetic disorder. In: Schollwöck, U., Richter, J., Farnell, D.J.J., Bishop, R.F. (eds.) Quantum Magnetism. Lecture Notes in Physics, vol. 645, p. 85. Springer, Berlin (2004)

57. Honecker, A., Schulenburg, J., Richter, J.: Magnetization plateaus in frustrated antiferromagnetic quantum spin models. J. Phys. Condens. Matter 16, S749 (2004)

58. Alicea, J., Chubukov, A.V., Starykh, O.A.: Quantum stabilization of the 1/3-magnetization plateau in $\mathrm{Cs}_{2} \mathrm{CuBr}_{4}$. Phys. Rev. Lett. 102, 137201 (2009)

59. Ono, T., Tanaka, H., Aruga Katori, H., Ishikawa, F., Mitamura, H., Goto, T.: Magnetization plateau in the frustrated quantum spin system $\mathrm{Cs}_{2} \mathrm{CuBr}_{4}$. Phys. Rev. B 67, 104431 (2003)

60. Fortune, N.A., Hannahs, S.T., Yoshida, Y., Sherline, T.E., Ono, T., Tanaka, H., Takano, Y.: Cascade of magnetic-field-induced quantum phase transitions in a spin-1/2 triangular-lattice antiferromagnet. Phys. Rev. Lett. 102, 257201 (2009)

61. Sakai, T., Nakano, H.: Critical magnetization behavior of the triangular- and kagome-lattice quantum antiferromagnets. Phys. Rev. B 83, 100405(R) (2011)

62. Seabra, L., Momoi, T., Sindzingre, P., Shannon, N.: Phase diagram of the classical Heisenberg antiferromagnet on a triangular lattice in an applied magnetic field. Phys. Rev. B 84, 214418 (2011)

63. Hotta, C., Nishimoto, S., Shibata, N.: Grand canonical finite size numerical approaches in one and two dimensions: real space energy renormalization and edge state generation. Phys. Rev. B 87, 115128 (2013)

64. Zhitomirsky, M.E.: Real-space perturbation theory for frustrated magnets: application to magnetization plateaus. J. Phys. Conf. Ser. 592, 012110 (2015)

65. Coletta, T., Zhitomirsky, M.E., Mila, F.: Quantum stabilization of classically unstable plateau structures. Phys. Rev. B 87, 060407 (2013)

66. Coletta, T., Tóth, T.A., Penc, K., Mila, F.: Semiclassical theory of the magnetization process of the triangular lattice Heisenberg model. Phys. Rev. B 94, 075136 (2016)

67. Villain, J., Bidaux, R., Carton, J.-P., Conte, R.: Order as an effect of disorder. J. Phys. (France) 41, 1263 (1980)

68. Shender, E.F.: Anti-ferromagnetic garnets with fluctuation-like interacting sub-lattices. Zh. Eksp. Teor. Fiz. 83, 326 (1982). [Sov. Phys. JETP 56, 178 (1982)]

69. Shirata, Y., Tanaka, H., Matsuo, A., Kindo, K.: Experimental realization of a spin-1/2 triangular-lattice Heisenberg antiferromagnet. Phys. Rev. Lett. 108, 057205 (2012)

70. Yamamoto, D., Marmorini, G., Danshita, I.: Quantum phase diagram of the triangular-lattice XXZ model in a magnetic field. Phys. Rev. Lett. 112, 127203 (2014)

71. Yamamoto, D., Marmorini, G., Danshita, I.: Erratum: Quantum phase diagram of the triangular-lattice XXZ model in a magnetic field. Phys. Rev. Lett. 112, 259901 (2014)

72. Starykh, O.A., Jin, W., Chubukov, A.V.: Phases of a triangular-lattice antiferromagnet near saturation. Phys. Rev. Lett. 113, 087204 (2014)

73. Sellmann, D., Zhang, X.-F., Eggert, S.: Phase diagram of the antiferromagnetic XXZ model on the triangular lattice. Phys. Rev. B 91, 081104(R) (2015)

74. Marmorini, G., Yamamoto, D., Danshita, I.: Umbrella-coplanar transition in the triangular XXZ model with arbitrary spin. Phys. Rev. B 93, 224402 (2016) 
75. Yamamoto, D., Ueda, H., Danshita, I., Marmorini, G., Momoi, T., Shimokawa, T.: Exact diagonalization and cluster mean-field study of triangular-lattice XXZ antiferromagnets near saturation. Phys. Rev. B 96, 014431 (2017)

Publisher's Note Springer Nature remains neutral with regard to jurisdictional claims in published maps and institutional affiliations.

\section{Affiliations}

D. J. J. Farnell ${ }^{1}$ (D R. F. Bishop ${ }^{2,3}$. J. Richter ${ }^{4,5}$

R. F. Bishop

raymond.bishop@manchester.ac.uk

J. Richter

Johannes.Richter@Physik.Uni-Magdeburg.DE

1 School of Dentistry, Cardiff University, Cardiff CF14 4XY, Wales, UK

2 School of Physics and Astronomy, The University of Manchester, Schuster Building, Manchester M13 9PL, UK

3 School of Physics and Astronomy, University of Minnesota, 116 Church Street SE, Minneapolis, MN 55455, USA

4 Institut für Physik, Otto-von-Guericke-Universität Magdeburg, 39016 Magdeburg, Germany

5 Max Planck Institute for the Physics of Complex Systems, 01187 Dresden, Germany 